\title{
Correlates of Listening Comprehension in L1 and L2: A Meta-analysis
}

\author{
Tuncay Karalık a * (D), Ali Merç a $†$ \\ a Anadolu University, Faculty of Education, Foreign Languages Education, Eskişehir, 26470, Turkey
}

Received 23 October 2018 | Received in revised form 04 March 2019 | Accepted 10 July 2019

\begin{abstract}
APA Citation:
Karalık, T. \& Merç, A. (2019). Correlates of listening comprehension in L1 and L2: A meta-analysis. Eurasian Journal of Applied Linguistics, 5(3), 353-383. Doi: 10.32601/ejal.651387
\end{abstract}

\begin{abstract}
The present meta-analysis aimed to explore the correlates of listening comprehension in first (L1) and second language (L2). In this regard, the overall average correlation scores, obtained from several primary studies retrieved from several databases, between linguistic (vocabulary size, vocabulary depth, syntactic knowledge), cognitive (working memory, metacognitive skills), and affective factors (anxiety and self-concept) and oral comprehension were measured. The results of the analysis revealed that in terms of strengths of association with listening comprehension, linguistic correlates were superior to cognitive correlates in L2 listening comprehension. There existed positive correlations with large effect sizes between vocabulary and grammar knowledge and listening comprehension while working memory yielded a positive correlation with a small effect size. Metacognitive skills, on the other hand, demonstrated a positive correlation with L2 listening comprehension with a large effect size. A similar pattern was observed in L1 listening comprehension with smaller strengths of association. Vocabulary and grammar knowledge yielded positive correlations with L1 listening comprehension and the effect size was medium while working memory weakly correlated with listening comprehension. An important distinction between L1 and L2 listening comprehension was observed in the affective domain. Anxiety and self-concept which were not reported to correlate with L1 listening comprehension were found to display large correlations with listening comprehension in L2. Overall, it was found that listening comprehension in both L1 and L2 is a multi-faceted process with different types of components involved.
\end{abstract}

(C) 2019 EJAL \& the Authors. Published by Eurasian Journal of Applied Linguistics (EJAL). This is an open-access article distributed under the terms and conditions of the Creative Commons Attribution license (CC BY-NC-ND) (http://creativecommons.org/licenses/by-nc-nd/4.0/).

Keywords: Listening comprehension; correlates of listening; meta-analysis; componential approach; L1 listening; L2 listening

\section{Introduction}

One crucial task language learners have to accomplish is comprehension of written and spoken texts (text comprehension henceforth) as there is plenty of input in language classes for language learners to process. Written and oral texts brought to language classrooms are important sources for providing input as language learners are expected to process these stimuli for their academic and linguistic development.

\footnotetext{
* tuncaykaralik@anadolu.edu.tr

$\dagger$ Corresponding author. Tel.: +90-222-3350580-3494

E-mail address: amerc@anadolu.edu.tr
} 
However, the rate or success of comprehension is not the same for each individual learner in the same learning environment. This is mostly because comprehension is a complex skill dependent on other component skills such as vocabulary knowledge (Chen, 2011; Choi, 2013), grammar knowledge (Shiotsu \& Weir, 2007; Yalin \& Wei, 2011), or background knowledge about the topic handled in the text (Lee, 2007; Leeser, 2007).

Therefore, the question of which factors are effective in explaining text comprehension is a difficult one to answer due to the complex nature of comprehension (Kim, 2015; Kim \& Cho, 2015). In other words, it may not be feasible to attribute success or failure in reading and listening comprehension to one component; rather it should be kept in mind that there is a complex relationship between many components at play.

The existence of component skills within a macro skill has important implications for teaching contexts. One significant implication is that language skills such as reading and listening comprehension have been viewed as a sum of component skills. Urquhart and Weir (1998), for example, suggested that the componential approach to reading comprehension aims at finding out whether certain constituents are present in the reading process. Similarly, Carr and Levy (1990) asserted that componential approaches to reading strive for identifying individual differences affecting reading and exploring their shared relationship and contributions to reading. Shiotsu and Weir (2007) also defined the goal of componential approach as "identifying possible explanatory skill factors or components involved in the reading process" (p. 99). They further elaborated the procedures in componential approaches suggesting that componential approach includes measuring learners' success in different components quantitatively and coming up with explanations about the effect of these components on the overall success in text comprehension based on the correlation scores between these measures and comprehension. In a nutshell, the first implication foresees that success in higher-order skills is dependent on success in lower order skills.

Another important implication is the diagnostic use of the componential approach in determining weaknesses of language learners in component skills and designing necessary interventions (Jeon \& Yamashita, 2014). In other words, studies with componential approach to text comprehension serve diagnostic purposes and help researchers and teachers prepare the necessary interventions to address comprehension difficulties resulting from deficiencies in component skills (Shiotsu \& Weir, 2007). In parallel with the first implication, success in higher-order skills depends on success in component skills. It has also been advocated that performance deficiencies in component skills reflect the areas to improve performance in complex skills.

The difficulty in detecting the factors that explain variance in reading and listening comprehension as the two complex skills has led researchers to adopt componential approach due to its inherent practicality in (a) determining what causes success or failure in certain skills and (b) designing the necessary interventions once the 
components are identified. As a result of these practicalities, componential research into reading and listening alike is thought to have much to offer for professionals in the field of language teaching since it can capture the complex nature of text comprehension and uncover the complex interaction among the components involved in the process.

\subsection{Conceptualization of text comprehension}

In line with the propositions of the componential approach, Kintsch (1988) proposed the Construction-Integration Model in order to capture the complex nature of text comprehension. In this model, comprehending texts, with no reference to any distinction between oral and written texts, is thought to consist of three distinct but related levels: surface level (vocabulary and grammar knowledge), propositional level (making meaning of sentences in discourse), and situation level (constructing meaning on prior knowledge). Figure 1 demonstrates the hierarchical relationship between the components present at each level.

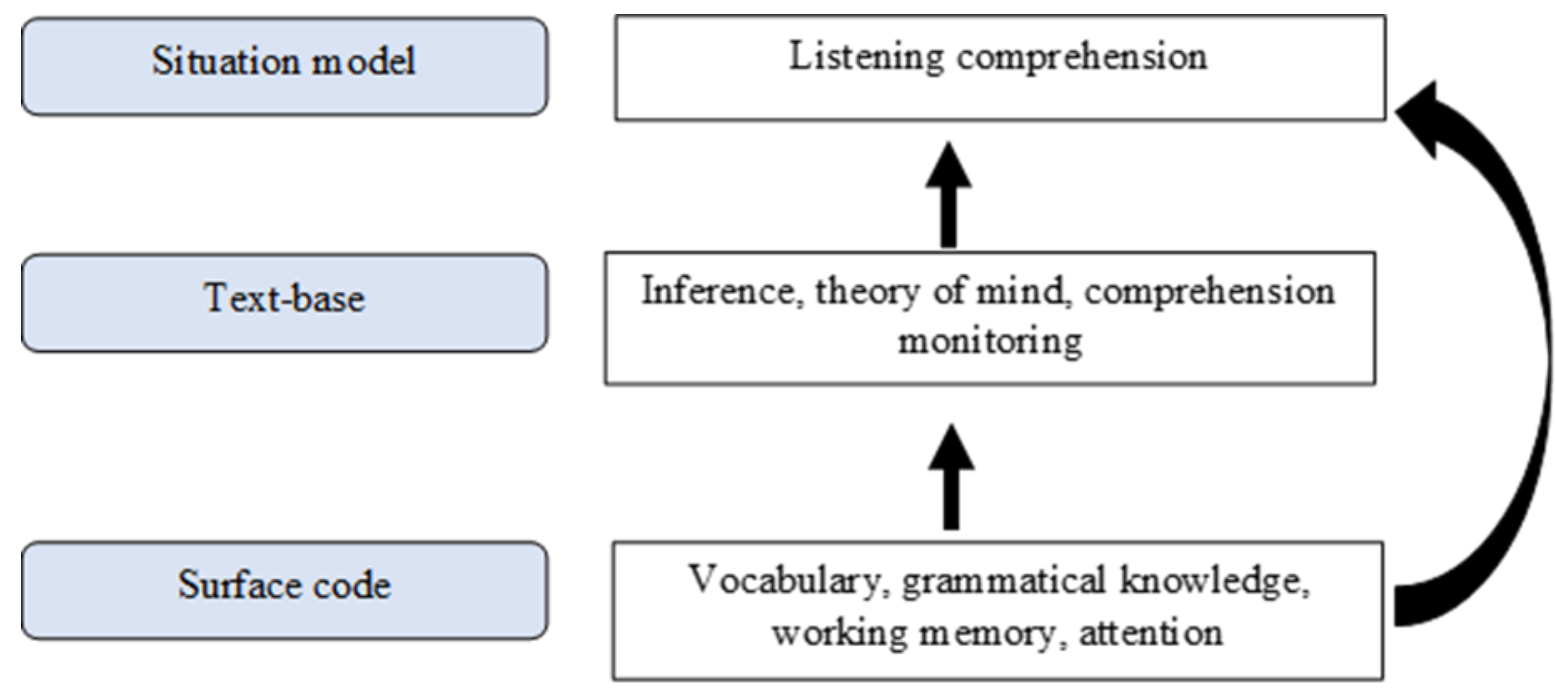

Figure 1. Conceptualization of text comprehension in Construction-Integration Model by Kintsch (1988)

As proposed in the model by Kintsch (1988), comprehension of texts is affected by learners' lower order skills such as working memory, grammatical knowledge, and vocabulary knowledge as well as their higher order skills such as inferencing ability and background knowledge about the topic handled in those texts. Similarly, as demonstrated in the model, lower level skills may affect success in higher-order skills. This idea underlines the complex relationship among the component skills and with text comprehension. Therefore, research into the factors leading to success or failure in comprehension should adopt a componential approach and take into account as many components involved in the process as possible without ignoring or overemphasizing certain components. The need for investigating the multifaceted nature of comprehension is well-reflected throughout the literature, especially in the reading research. For example, a comprehensive meta-analysis by Jeon and 
Yamashita (2014) presented results from several studies on correlates of reading comprehension including high evidence correlates, those which were frequently addressed, such as vocabulary knowledge, grammar knowledge, L2 decoding and L1 reading comprehension; and low evidence correlates, those investigated less frequently, such as working memory, L2 orthographic knowledge, L2 morphological knowledge, L2 phonological awareness and metacognition. The study is an important attempt to systematically collect evidence of the multi-faceted nature of text comprehension and present the strengths of associations between cognitive and linguistic components and comprehension. Listening comprehension is also a similar process of decoding incoming messages. However, to date there has not been a metaanalytic study to provide robust evidence of the multi-faceted nature of listening comprehension.

As text comprehension is a multi-faceted process, researchers have to handle several components separately or together, which brings about many independent studies addressing different components. Consequently, a systematic collection of these independent studies is needed for a comprehensive panorama of the skills involved in comprehension. Therefore, the current meta-analysis aims to investigate the correlates of listening comprehension by answering the following questions:

1. What are the components of listening and their strengths of association with listening comprehension?

2. Is there a difference between L2 listening and L2 reading with regard to their correlates?

3. Is there a difference between L1 and L2 listening comprehension with regard to their correlates?

A systematic analysis of studies on the relationship between component skills of listening comprehension could contribute significantly to our understanding of the complex relationship between these subskills and listening comprehension. Metaanalysis is defined as a kind of statistical procedure employed to calculate the mean and variance of effects sizes obtained from a collection of primary studies in a specific area (Plonsky \& Oswald, 2012). In addition to this narrower definition, Plonksy and Oswald (2012) provide a broader definition which includes not only the statistical computations but also "the expert's understanding, translation, and communication of the research studies and samples involved, along with the best that theory has to offer across studies and beyond those studies" (p. 275). In other words, meta-analysis requires comprehensive search for studies, mostly quantitative studies, from different sources and combining the results from these studies to come up with a theoretical understanding of the issue at hand. One important advantage of meta-analysis is that results come from many individual primary studies, and this, in turn, provides a more panoramic view of the issue handled. In language teaching research, such an analysis can act as a diagnostic step to improve a certain skill by uncovering the commonly studied correlates of that specific skill. Therefore, the current meta-analysis aims to find out the subcomponents of listening comprehension and explore how successful 
these component skills can account for variance among language learners in terms of listening comprehension.

\section{Review of the Components of Listening Comprehension}

Like reading comprehension, listening comprehension is a skill that should be researched with a componential approach since several different factors determine learners' success or failure. As such, several studies have been devoted to investigating the components of listening comprehension which can be classified as linguistic (vocabulary and grammar knowledge), cognitive (working memory and metacognitive strategy use) and affective (anxiety and motivation).

\subsection{Linguistic components}

Defined as knowledge of forms and meanings of words (Nation, 2001) in its simplest sense, vocabulary knowledge is one of the most important components of all language skills. The role of vocabulary knowledge in linguistic is nearly taken for granted and one of the main goals of language teaching programs is to build as large vocabulary repertoire among language learners as possible. Several attempts have been made to investigate how to teach vocabulary through vocabulary tasks in short periods of language teaching programs (Beal, 2007; Keating, 2008). The main motive behind such studies has always been the idea that vocabulary knowledge is at the heart of language learning and it is essential to have sufficient amount of vocabulary knowledge either to express oneself or decode the incoming stimuli. Several studies addressed the importance of vocabulary knowledge in writing (Al Seyabi \& Tuzlukova, 2014; Huy, 2015; Sawaki, Quinlan, \& Lee, 2013) underlining that deficiencies in vocabulary knowledge result in learners having difficulties in arranging and conveying ideas to the readers. Similar difficulties are reported to emerge during spoken communication due to lack of vocabulary knowledge (Khotimah, 2014; Pérez Manzanilla \& Diaz Cabrera, 2014; Tahir, 2015). Taken together with studies underlining the strong relationship with vocabulary knowledge and reading comprehension, the results of these studies highlight the importance of vocabulary knowledge in production as well comprehension. Strong correlations between comprehension and vocabulary knowledge have also been obtained in L2 listening (Andringa, Olsthoorn, Van Beuningen, Schoonen, \& Hulstijn, 2012; Bonk, 2000; Matthews \& Cheng, 2015; Oh, 2016; Stæhr, 2008; Teng, 2014; Tighe, Spencer, \& Schatschneider, 2015). Higher levels of vocabulary knowledge have been associated with higher levels of listening comprehension and similarly deficiencies in lexical knowledge resulted in lack of comprehension.

Another important component of language skills is grammar knowledge. Grammar knowledge can be defined as knowledge about some morphosyntactic features as tense, subject-verb agreement, aspect and articles (Jeon \& Yamashita, 2014). As in the case of vocabulary knowledge, the role of grammar knowledge in linguistic development in all skills has been highlighted. The teaching of grammar has been 
found effective in promoting writing skill among L2 learners by helping them organize their ideas and express themselves more clearly (Jones, Myhill, \& Bailey, 2013). However, there is lack of evidence to advocate that grammar knowledge is vital for speaking fluency. The research up to date has usually acknowledged the important role grammar knowledge plays in comprehension of the incoming stimuli, both written and oral, rather than in production. As is the case in L2 reading research, grammar knowledge has been found to be strong correlating with L2 listening comprehension (Oh, 2016; Sağlam, 2014). However, a closer look into the existing literature clearly shows that there is a great scarcity of studies into the relationship between grammar knowledge and L2 listening comprehension, which calls for further studies into the issue.

\subsection{Cognitive components}

An oft-discussed question in L2 reading literature has been whether reading comprehension is a language or a reading problem since Alderson (1984) asked it. His concern was to determine if language learners differed from each other in terms of L2 reading comprehension due to language-neutral cognitive differences such as working memory and metacognitive strategies used or language-specific linguistic factors such as vocabulary and grammar knowledge. The existing literature on L2 reading comprehension seems to have resolved the question in favor of linguistic factors. However, when we turn our attention to L2 listening comprehension studies, due to low empirical accumulation, we are far from a conclusive answer to the question whether L2 listening comprehension is a language or listening problem. Considering the existing evidence, working memory is not a significant contributor to L2 listening comprehension since the reported correlation is weak (Andersson, 2010; Andringa et al., 2012; Brunfaut \& Révézs, 2015; Call, 1985; Tighe et al., 2015). On the other hand, metacognition has been reported to yield weak to strong correlations with L2 listening comprehension (Amin, Aly, \& Mohammed, 2015; Kassem, 2015; Sağlam, 2014).

\subsection{Affective components}

In educational settings, specifically L2 learning contexts, students don't come to the class as human beings with only cognitive and linguistic differences affecting the process of language learning but they also bring to the classroom affective differences. Krashen (1985) for example suggests that in order for comprehensible input to be received by the learner, affective barriers (also referred as affective filter) should be overcome. In line with this suggestion, scholars have often looked into the effects of motivation and anxiety on language learning. Defined by psychologists as a state of apprehension and a fear that is indirectly associated with an object (Scovel, 1978), anxiety has been shown to have debilitating effects on language learners in speaking (Woodrow, 2006; Zhiping, 2013), in writing (Cheng, 2002; Cheng, Horwitz, \& Schallert, 1999); in reading (Saito, Garza, \& Horwitz, 1999; Sellers, 2000; Zin \& Rafik-Galea, 2010). We witness a similar case in L2 listening research with anxiety 
correlating negatively with listening comprehension scores (Atasheneh \& Izadi, 2012; Brunfaut \& Révész, 2015; Elkhafaifi, 2005; Serraj \& Noordin, 2013). The studies demonstrated that when anxiety levels increased, language learners have difficulties in oral comprehension. Put it differently, those learners with lower levels of anxiety performed better in comprehension than those suffering from high levels of anxiety. Considering the huge effect of anxiety on listening comprehension along with a relatively low number of studies conducted so far, it is a must for further studies to address this factor along with other components.

On the opposite side of the affective domain, there is a motivation factor which contributes to language learning in the reverse direction. Motivation has been reported to yield a moderate correlation with L2 listening comprehension (Tafaghodtari \& Vandergrift, 2008).

\section{Method}

\subsection{Literature Review and Inclusion of Studies}

The present inquiry covered studies that adopted a componential approach to text comprehension with the components and text comprehension measured quantitatively. In other words, the scope of the present analysis is the studies which reported correlational values between components and listening comprehension

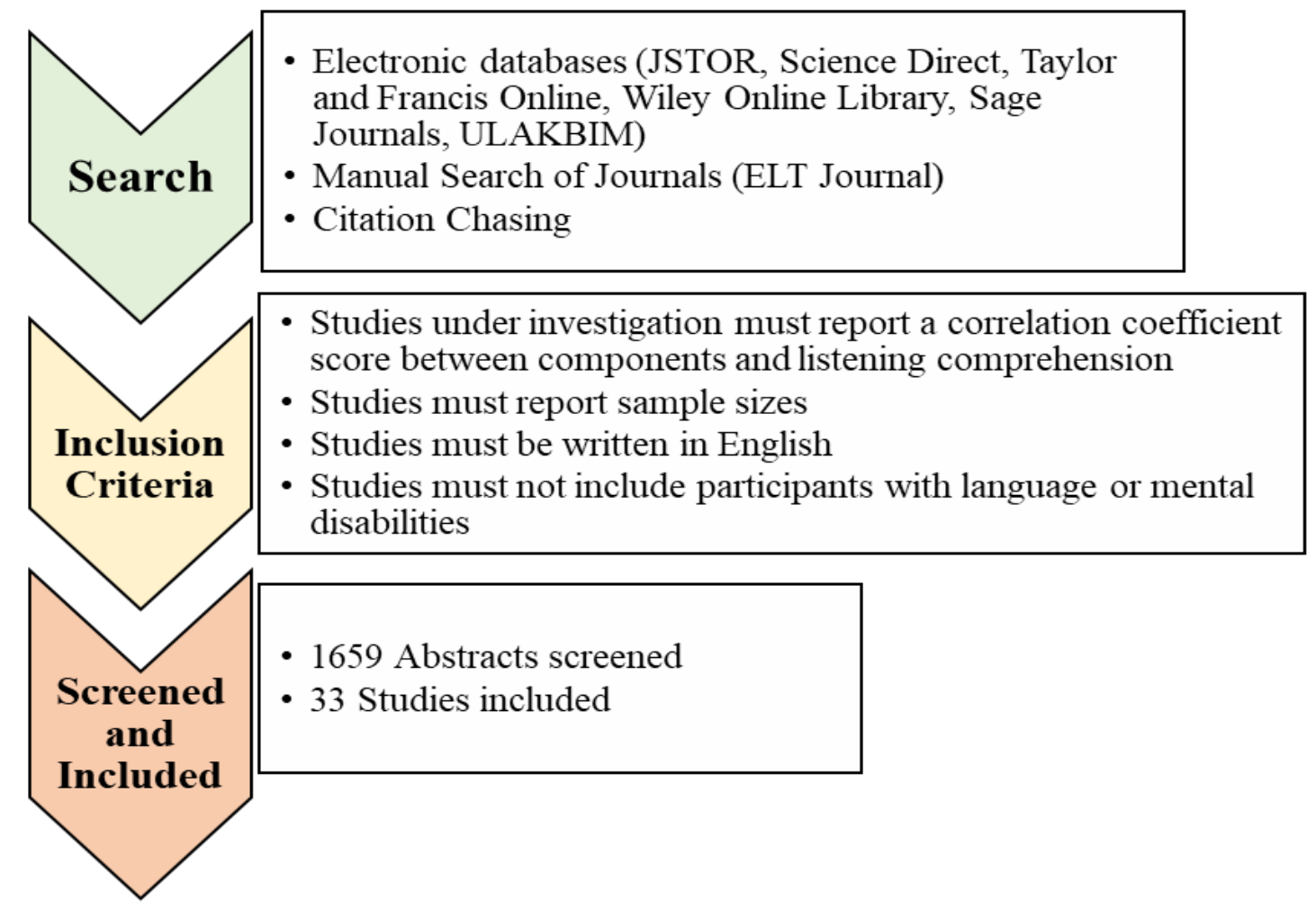

Figure 2. Steps of search and inclusion of studies 
scores. The steps of locating the relevant studies are presented in Figure 2.

First, as Figure 2 shows, a number of electronic databases and journals were screened to locate relevant inquiries into the components of listening comprehension. In order to obtain as many articles into listening comprehension as possible, no time range was set. The electronic databases and the journal were searched using the terms "listening comprehension", "oral comprehension", "components", "listening skill" so as not to limit the search for specific components. Another precaution for not limiting the results was that the entries were searched not only in the titles but also in the abstracts. Moreover, studies were located through citation chasing in review articles. As a result of the searching procedure, 1659 abstracts were screened and the articles which seemed to use a correlational design were retrieved from the databases. Among these studies, only those which included samples without any mental or linguistic disorders were analyzed further. The studies that recruited participants with such disorders were excluded from the analysis, as the results obtained from participants with special needs would discourage us to generalize the results. Finally, only the studies which reported sample sizes and correlation results were included in the present meta-analysis. The studies with pre and pots-test and/or experimental design were excluded from the analysis since the main motive of the study was to find out the strengths of associations between the component skills and listening comprehension. Furthermore, the studies reporting duplicate samples were excluded from the analysis. Table 1 presents a brief account of the studies (participants, variables under investigation, instruments, and results).

\subsection{Analysis of the studies}

The studies included in the meta-analysis were first coded for the status of the language on which listening comprehension was measured since L1 and L2 listening comprehension were handled separately. Once the coding was completed, the studies that used a longitudinal design and reported correlation coefficients at different time points were determined. Only one correlation coefficient was reported among different time points (e.g., Jeon and Yamashita, 2014), the one which was closer to the rest of the studies in terms of effect size in the present case. As for studies which reported using more than one instrument to measure a specific variable, the average score from the instruments was reported. Once the coding of the studies as L1 or L2 was complete and when the correlational values were determined, the studies were entered into the Comprehensive Meta-Analysis software. The correlation scores between the components and listening comprehension were calculated as well as their $95 \%$ confidence of interval for the association between the variables and listening comprehension. In order to interpret correlation coefficient sizes, discipline-specific benchmarks proposed by Plonksy and Oswald (2014) were used after careful analysis of primary and meta-analytic studies in the field of second language acquisition. Plonsky and Oswald (2014) suggest "rs close to .25 be considered small, .40 medium, and .60 large" (p. 889). 


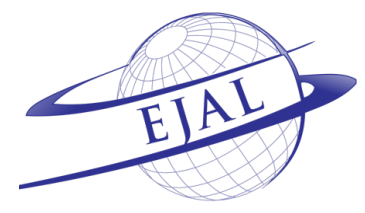

Available online at www.ejal.eu

http://dx.doi.org/10.32601/ejal.651387

Eurasian Journal of Applied Linguistics, 5(3), 353-383

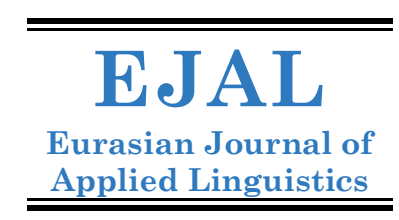

Table 1. Overview of the studies included in the meta-analysis

\begin{tabular}{|c|c|c|c|c|}
\hline & Participants & Components & Instruments & Results \\
\hline Adams et al. (1999) & 66 English Children & $\begin{array}{l}\text { Working memory, vocabulary } \\
\text { comprehension }\end{array}$ & $\begin{array}{l}\text { Comprehension sub-scale of Reynell } \\
\text { Developmental Language Scales, word span } \\
\text { task, The British Picture Vocabulary Scale }\end{array}$ & $\begin{array}{l}\text { Working memory }(r=.30) \\
\text { Vocabulary comprehension }(r=.65)\end{array}$ \\
\hline Amin et al. (2011) & $\begin{array}{l}80 \text { Egyptian learners of } \\
\text { English }\end{array}$ & Strategic listening & $\begin{array}{l}\text { Listening test, strategic listening } \\
\text { questionnaire, }\end{array}$ & Strategic listening $(r=.80)$ \\
\hline Andersson (2010) & $\begin{array}{l}95 \text { Swedish learners of } \\
\text { English }\end{array}$ & Working memory & Listening comprehension task, word span task & Working memory $(\mathrm{r} .=.28)$ \\
\hline Andringa et al. (2012) & $\begin{array}{l}121 \text { native and } 113 \text { non- } \\
\text { native speakers of Dutch }\end{array}$ & $\begin{array}{l}\text { Vocabulary, working memory, } \\
\text { grammatical processing }\end{array}$ & $\begin{array}{l}\text { Dutch State Exam of Listening Proficiency, } \\
\text { Receptive vocabulary test, Digit and non-word } \\
\text { span tasks, Sentence initial acceptability task }\end{array}$ & $\begin{array}{l}\underline{\text { Native speakers }} \\
\text { Vocabulary }(r=.35) \\
\text { Grammatical processing }(r=.29 \\
\text { Working memory }(r=.37) \\
\text { Non-native speakers } \\
\text { Vocabulary }(r=.68) \\
\text { Grammatical processing }(r=.77) \\
\text { Working memory }(r=.21)\end{array}$ \\
\hline Atasheneh \& Izadi (2012) & $\begin{array}{l}\text { 60 Iranian learners of } \\
\text { English }\end{array}$ & $\begin{array}{l}\text { Foreign language learning } \\
\text { anxiety }\end{array}$ & $\begin{array}{l}\text { Two listening tests of Main Street } \\
\text { Series, foreign Language Class Anxiety }\end{array}$ & Foreign language learning anxiety $(r=-69)$ \\
\hline Bonk (2000) & $\begin{array}{l}59 \text { Japanese learners of } \\
\text { English }\end{array}$ & Lexical familiarity & Four listening passages, Dictation task & Lexical familiarity $(r=.44)$ \\
\hline Brunfaut \& Révész (2015) & $\begin{array}{l}93 \text { Non-native learners of } \\
\text { English }\end{array}$ & $\begin{array}{l}\text { Working memory, listening } \\
\text { anxiety }\end{array}$ & $\begin{array}{l}\text { Passage completion task, span tasks, listening } \\
\text { anxiety scale }\end{array}$ & $\begin{array}{l}\text { Working memory }(r=.29) \\
\text { Listening anxiety }(r=-54)\end{array}$ \\
\hline Call (1985) & $\begin{array}{l}41 \text { Arabic/Spanish learners } \\
\text { of English }\end{array}$ & Working memory & $\begin{array}{l}\text { Michigan Test of Aural Comprehension, } \\
\text { random word repetition task }\end{array}$ & Working memory $(r=.39)$ \\
\hline
\end{tabular}




\begin{tabular}{|c|c|c|c|c|}
\hline Elkhafaifi (2005) & $\begin{array}{l}233 \text { learners of Arabic from } \\
\text { several backgrounds }\end{array}$ & $\begin{array}{l}\text { Foreign language listening } \\
\text { anxiety }\end{array}$ & $\begin{array}{l}\text { Listening grades, foreign language listening } \\
\text { anxiety scale }\end{array}$ & Foreign language listening anxiety $(r=.-70)$ \\
\hline $\begin{array}{l}\text { Florit, Roch, Altoè, \& Levorato } \\
\text { (2009) }\end{array}$ & 84 Italian children & $\begin{array}{l}\text { Vocabulary knowledge, working } \\
\text { memory }\end{array}$ & $\begin{array}{l}\text { Test for listening comprehension, Peabody } \\
\text { Picture Vocabulary Test, word span task }\end{array}$ & $\begin{array}{l}\text { Vocabulary knowledge }(r=40) \\
\text { Working memory }(r=.38)\end{array}$ \\
\hline $\begin{array}{l}\text { Fracasso, Bangs, \& Binder } \\
\text { (2016) }\end{array}$ & $\begin{array}{l}55 \text { Adult speakers of } \\
\text { English as a second } \\
\text { language }\end{array}$ & Morphological awareness & $\begin{array}{l}\text { Listening comprehension test, } \\
\text { derivation/suffix choice/production tasks }\end{array}$ & Morphological awareness $(r=.76)$ \\
\hline Kassem (2015) & $\begin{array}{l}\text { 84 Egyptian learners of } \\
\text { English }\end{array}$ & Listening strategy & $\begin{array}{l}\text { Listening sub-test of TOEFL, listening } \\
\text { strategy questionnaire }\end{array}$ & Listening strategy $(r=.62)$ \\
\hline Kim (2015) & 148 Korean children & $\begin{array}{l}\text { Vocabulary knowledge, syntactic } \\
\text { knowledge, working memory }\end{array}$ & $\begin{array}{l}\text { Peabody Picture Vocabulary Test, Narrative } \\
\text { listening comprehension test, syntactic } \\
\text { knowledge task, listening span task }\end{array}$ & $\begin{array}{l}\text { Vocabulary knowledge }(r=.42) \\
\text { Syntactic knowledge }(r=.46) \\
\text { Working memory }(r=.17)\end{array}$ \\
\hline Kim (2016) & 201 Korean children & $\begin{array}{l}\text { Working memory, vocabulary } \\
\text { knowledge, grammar knowledge, } \\
\text { inference skills, }\end{array}$ & $\begin{array}{l}\text { Two listening texts, listening span task, } \\
\text { expressive vocabulary task, grammatical } \\
\text { knowledge task, inference task }\end{array}$ & $\begin{array}{l}\underline{\text { Text } 1} \\
\text { Working memory }(r=.43) \\
\text { Vocabulary knowledge }(r=.40) \\
\text { Grammar knowledge }(r=.45) \\
\text { Inference skills }(r=.50) \\
\underline{\text { Text } 2} \\
\text { Working memory }(r=.31) \\
\text { Vocabulary knowledge }(r=.30) \\
\text { Grammar knowledge }(r=.31) \\
\text { Inference skills }(r=.33)\end{array}$ \\
\hline Kim \& Philips (2014) & 156 children & Vocabulary knowledge & Test of narrative language, Woodcock-Johnson & Vocabulary knowledge $(r=.59)$ \\
\hline Kök (2017) & $\begin{array}{l}44 \text { Turkish learners of } \\
\text { English }\end{array}$ & Listening strategy & $\begin{array}{l}\text { Listening sub-test of IELTS, listening } \\
\text { comprehension strategy use inventory }\end{array}$ & $\begin{array}{l}\text { Listening comprehension strategy use } \\
(r=.86)\end{array}$ \\
\hline
\end{tabular}


Lepola, Lynch, Laakkonen, Silvén, \& Niemi (2012)
130 Finnish children

Phonological awareness,

vocabulary knowledge, sentence memory, inference skills
Text comprehension test, rhyme and

alliteration tasks, word definition test, sentence repetition task, picture book reviewing

\section{Time}

Vocabulary knowledge $(r=.45)$

Phonological awareness $(r=.31)$

Sentence memory $(r=.50)$

Inference skills $(r=.39)$

Time 2

Vocabulary knowledge $(r=.53)$

Sentence memory $(r=.31)$

Inference skills $(r=.51)$

Time 3

Vocabulary knowledge $(r=.45)$

Sentence memory $(r=.35)$

Inference skills $(r=.54)$

\begin{tabular}{|c|c|c|c|c|}
\hline Matthews \& Cheng (2015) & $\begin{array}{l}167 \text { Chinese learners of } \\
\text { English }\end{array}$ & Vocabulary knowledge & $\begin{array}{l}\text { Listening sub-test of IELTS, a word } \\
\text { recognition test }\end{array}$ & Vocabulary knowledge $(r=.73)$ \\
\hline Oh (2016) & $\begin{array}{l}75 \text { Korean learners of } \\
\text { English }\end{array}$ & $\begin{array}{l}\text { Vocabulary knowledge, grammar } \\
\text { knowledge }\end{array}$ & $\begin{array}{l}\text { Listening parts of the mid-term and final } \\
\text { exams, auditory vocabulary test, sentence } \\
\text { completion task }\end{array}$ & $\begin{array}{l}\text { Vocabulary knowledge }(r=.59) \\
\text { Grammar knowledge }(r=.55)\end{array}$ \\
\hline Rahimi \& Abedini (2009) & $\begin{array}{l}\text { 61 Iranian learners of } \\
\text { English }\end{array}$ & Self-efficacy & $\begin{array}{l}\text { Listening sub-test of TOEFL, Self-efficacy } \\
\text { questionnaire }\end{array}$ & Self-efficacy $(r=.78)$ \\
\hline Sağlam (2014) & $\begin{array}{l}73 \text { Turkish learners of } \\
\text { English }\end{array}$ & $\begin{array}{l}\text { Grammar knowledge, vocabulary } \\
\text { knowledge, listening strategy use }\end{array}$ & $\begin{array}{l}\text { TOEFL listening comprehension test, TOEFL } \\
\text { structure test, vocabulary levels test }\end{array}$ & $\begin{array}{l}\text { Vocabulary knowledge }(r=.71) \\
\text { Grammar knowledge }(r=.70) \\
\text { Strategy use }(r=.128)\end{array}$ \\
\hline Serraj \& Noordin (2013) & $\begin{array}{l}210 \text { Iranian learners of } \\
\text { English }\end{array}$ & $\begin{array}{l}\text { Foreign language listening } \\
\text { anxiety }\end{array}$ & $\begin{array}{l}\text { IELTS listening practice test, foreign } \\
\text { language listening anxiety scale }\end{array}$ & $\begin{array}{l}\text { Foreign language listening anxiety }(r=- \\
414)\end{array}$ \\
\hline Stæhr (2008) & $\begin{array}{l}88 \text { Danish learners of } \\
\text { English }\end{array}$ & Vocabulary size & $\begin{array}{l}\text { Vocabulary Levels Test, listening } \\
\text { comprehension test }\end{array}$ & Vocabulary size $(r=.69)$ \\
\hline Stæhr (2009) & $\begin{array}{l}115 \text { Danish learners of } \\
\text { English }\end{array}$ & $\begin{array}{l}\text { Vocabulary size, depth of } \\
\text { vocabulary knowledge }\end{array}$ & $\begin{array}{l}\text { Vocabulary Levels Test, depth of vocabulary } \\
\text { knowledge test, listening comprehension test }\end{array}$ & $\begin{array}{l}\text { Vocabulary size }(r=.70) \\
\text { Vocabulary depth }(r=.65)\end{array}$ \\
\hline
\end{tabular}




\begin{tabular}{|c|c|c|c|c|}
\hline Tabrizi \& Saeidi (2015) & $\begin{array}{l}90 \text { Iranian learners of } \\
\text { English }\end{array}$ & Listening self-efficacy & $\begin{array}{l}\text { TOEFL listening practice test, listening self- } \\
\text { efficacy beliefs questionnaire }\end{array}$ & Listening self-efficacy $(r=.56)$ \\
\hline $\begin{array}{l}\text { Tafaghodtari \& Vandergrift } \\
\text { (2008) }\end{array}$ & $\begin{array}{l}115 \text { Iranian learners of } \\
\text { English }\end{array}$ & $\begin{array}{l}\text { Metacognitive awareness, } \\
\text { language learning motivation }\end{array}$ & $\begin{array}{l}\text { Listening sub-test of the institutional } \\
\text { proficiency test, MALQ, language learning } \\
\text { motivation orientation scale }\end{array}$ & $\begin{array}{l}\text { Metacognitive awareness }(r=.57) \\
\text { Motivation }(r=.46)\end{array}$ \\
\hline Teng (2014) & $\begin{array}{l}88 \text { Chinese learners of } \\
\text { English }\end{array}$ & $\begin{array}{l}\text { Vocabulary size, depth of } \\
\text { vocabulary knowledge }\end{array}$ & $\begin{array}{l}\text { IELTS listening comprehension test, } \\
\text { vocabulary size test, depth of vocabulary } \\
\text { knowledge test }\end{array}$ & $\begin{array}{l}\text { Vocabulary size }(r=.86) \\
\text { Vocabulary depth }(r=.91)\end{array}$ \\
\hline Teng (2016) & $\begin{array}{l}88 \text { Chinese learners of } \\
\text { English }\end{array}$ & $\begin{array}{l}\text { Vocabulary size, depth of } \\
\text { vocabulary knowledge }\end{array}$ & $\begin{array}{l}\text { IELTS academic listening comprehension test, } \\
\text { vocabulary size test, depth of vocabulary } \\
\text { knowledge test }\end{array}$ & $\begin{array}{l}\text { Vocabulary size }(r=.70) \\
\text { Vocabulary depth }(r=.75)\end{array}$ \\
\hline Tighe et al. (2015) & $\begin{array}{l}2153^{\text {rd }} \text { graders } \\
1887^{\text {th }} \text { graders } \\
18210^{\text {th }} \text { graders from } \\
\text { several backgrounds }\end{array}$ & $\begin{array}{l}\text { Working memory, reasoning } \\
\text { skills, vocabulary }\end{array}$ & $\begin{array}{l}\text { Listening sub-test of Florida Comprehensive } \\
\text { Assessment Test, competing language } \\
\text { processing task, Wechsler Abbreviated Scale } \\
\text { of Intelligence (different subtests for reasoning } \\
\text { and vocabulary) }\end{array}$ & $\begin{array}{l}\underline{3^{r d} \text { graders }} \\
\text { Working memory }(r=.40) \\
\text { Reasoning skills }(r=.44) \\
\text { Vocabulary knowledge }(r=.62) \\
\underline{7^{\text {th }} \text { graders }} \\
\text { Working memory }(r=.32) \\
\text { Reasoning skills }(r=.39) \\
\text { Vocabulary knowledge }(r=.57) \\
\underline{10^{\text {th }} \text { graders }} \\
\text { Working memory }(r=.15) \\
\text { Reasoning skills }(r=.36) \\
\text { Vocabulary knowledge }(r=.46)\end{array}$ \\
\hline Vandergrift \& Baker (2015) & $\begin{array}{l}157 \text { learners of English in a } \\
\text { Canadian context }\end{array}$ & $\begin{array}{l}\text { Vocabulary knowledge, working } \\
\text { memory, metacognition, }\end{array}$ & $\begin{array}{l}\text { English listening comprehension test, Peabody } \\
\text { Picture Vocabulary Test, WTMB-C (Working } \\
\text { Memory Test Battery for Children), MALQ }\end{array}$ & $\begin{array}{l}\text { Vocabulary knowledge }(r=.51) \\
\text { Working memory }(r=.20) \\
\text { Metacognition }(r=.23)\end{array}$ \\
\hline $\begin{array}{l}\text { Vulchanova, Foyn, Nilsen, \& } \\
\text { Sigmundsson (2014) }\end{array}$ & $\begin{array}{l}84 \text { Norwegian learners of } \\
\text { English }\end{array}$ & $\begin{array}{l}\text { Working memory, vocabulary } \\
\text { knowledge }\end{array}$ & $\begin{array}{l}\text { English listening comprehension test, working } \\
\text { memory test battery for children, Peabody } \\
\text { Picture Vocabulary Test }\end{array}$ & $\begin{array}{l}\text { Working memory }(r=.40) \\
\text { Vocabulary knowledge }(r=.46)\end{array}$ \\
\hline
\end{tabular}




\begin{tabular}{|c|c|c|c|c|}
\hline Wang \& Treffers-Daller (2017) & $\begin{array}{l}172 \text { Chinese learners of } \\
\text { English }\end{array}$ & $\begin{array}{l}\text { Vocabulary knowledge, } \\
\text { Metacognition }\end{array}$ & $\begin{array}{l}\text { Listening subtest of CET-4, Vocabulary size } \\
\text { test, MALQ }\end{array}$ & $\begin{array}{l}\text { Vocabulary knowledge }(r=.44) \\
\text { Metacognition }(r=.19)\end{array}$ \\
\hline $\begin{array}{l}\text { Wolfgramm, Suter, \& Göksel } \\
\text { (2016) }\end{array}$ & $\begin{array}{l}354 \text { Swiss learners of } \\
\text { German }\end{array}$ & $\begin{array}{l}\text { Concentration, working memory, } \\
\text { vocabulary knowledge, self- } \\
\text { concept }\end{array}$ & $\begin{array}{l}\text { Klassencockpit Listening Comprehension } \\
\text { Test, Harmos Listening Comprehension Test, } \\
\text { Test Battery for the Assessment of } \\
\text { Concentration, Letter-Number Sequencing } \\
\text { task, vocabulary subtest of Klassencockpit } \\
\text { test, Differential Vocational Self-concept Grid }\end{array}$ & $\begin{array}{l}\text { Klassencockpit } \\
\text { Concentration }(r=.26) \\
\text { Working memory }(r=.14) \\
\text { Vocabulary knowledge }(r=.459) \\
\text { Self-concept }(r=.30) \\
\underline{\text { Harmo } S} \\
\text { Concentration }(r=.39) \\
\text { Working memory }(r=.35) \\
\text { Vocabulary knowledge }(r=.48) \\
\text { Self-concept }(r=.35)\end{array}$ \\
\hline
\end{tabular}




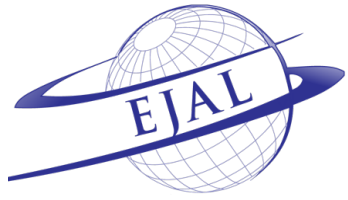

Available online at www.ejal.eu

http://dx.doi.org/10.32601/ejal.651387

Eurasian Journal of Applied Linguistics, 5(3), 353-383

EJAL

Eurasian Journal of Applied Linguistics

\section{Results}

\subsection{Correlates of L1 listening comprehension}

The number of independent samples, overall correlation coefficients and 95\% confidence of intervals of the correlates of L1 listening comprehension are presented in Table 2. If the CI value is higher than 0 , the correlation is significantly different from 0 .

Table 2. Overall correlations between the components and L1 listening comprehension

\begin{tabular}{llll}
\hline Component & $\mathrm{N}$ & $r[95 \% \mathrm{CI}]$ & $I^{2}$ \\
\hline Vocabulary Knowledge & 7 & $.45[.35-.54]^{* *}$ & 68.635 \\
Grammar Knowledge & 3 & $.36[.25-.46]^{* *}$ & 41.800 \\
Phonological Awareness & 2 & $.57[-.01-.86]$ & 94.063 \\
Morphological Awareness & & $.31[.24-.37]^{* *}$ & 0.000 \\
Working Memory & 6 & &
\end{tabular}

As Table 2 shows, most of the studies addressed vocabulary knowledge followed by working memory and grammar knowledge. The effect sizes ranged from medium to weak.

\subsubsection{Linguistic correlates of L1 listening comprehension}

\subsubsection{Vocabulary knowledge and L1 listening comprehension}

Vocabulary knowledge was the most commonly investigated correlate of L1 listening comprehension. Seven different studies with seven independent samples and a total of 906 participants reported correlation coefficients for vocabulary knowledge and L1 listening comprehension (mean sample size $=129.4, S D=45.33$, range $=66$ 201). The participants had several L1s: Finnish (1 Sample), Dutch (1 Sample), Korean (2 Samples), English (2 Samples), and Italian (1 Sample) and their ages ranged from kindergarten to adults. The individual and overall correlations reported in the studies are demonstrated in Figure 3 (the squares indicate individual correlation coefficients and the diamond represents the overall mean correlation).

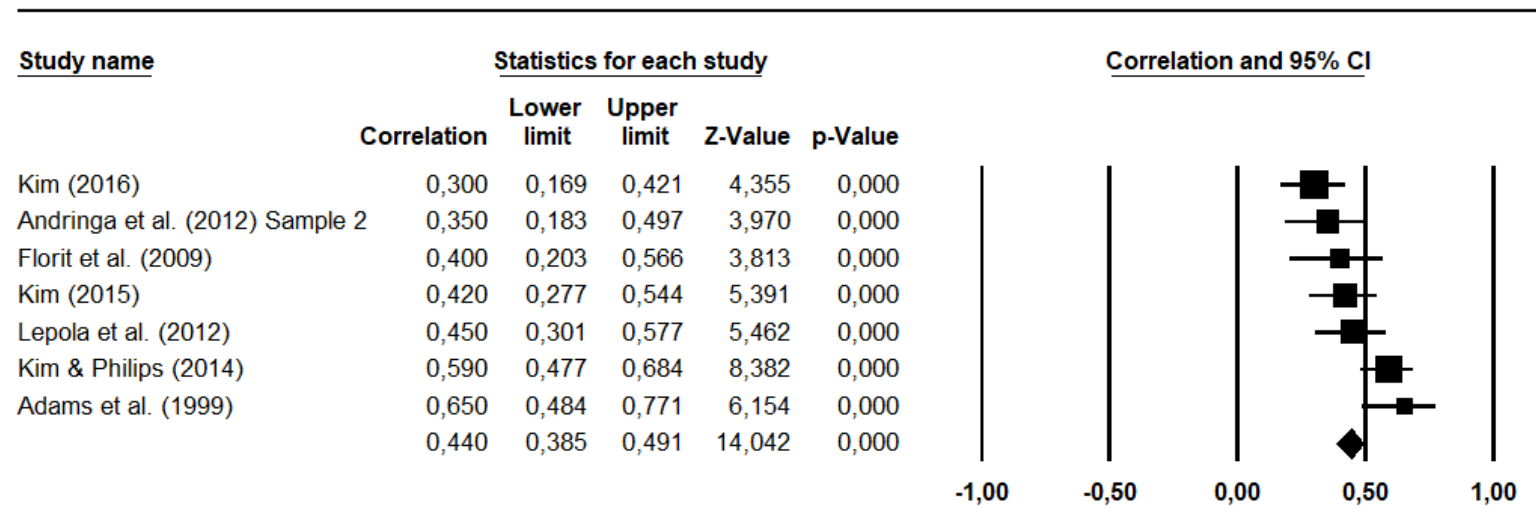

Figure 3. Reported correlations between vocabulary knowledge and listening comprehension 
The correlation between L1 vocabulary knowledge and L1 listening comprehension was found to be positive and the effect size was medium ( $r=.45,95 \%$ CI [.35-.54]) and significant $(p<.001)$. This finding suggests that vocabulary knowledge plays an important role in predicting L1 listening success. Higher vocabulary knowledge meant higher listening comprehension scores and similarly, lower vocabulary knowledge brought about lower comprehension scores.

\subsubsection{Grammar knowledge and L1 listening comprehension}

Grammar knowledge was addressed in three studies with three independent samples. The total number of participants in the studies were 470 (mean sample size $=156.6, S D=40.69$, range $=121-201)$. Figure 4 shows the overall correlation coefficients obtained in these studies.

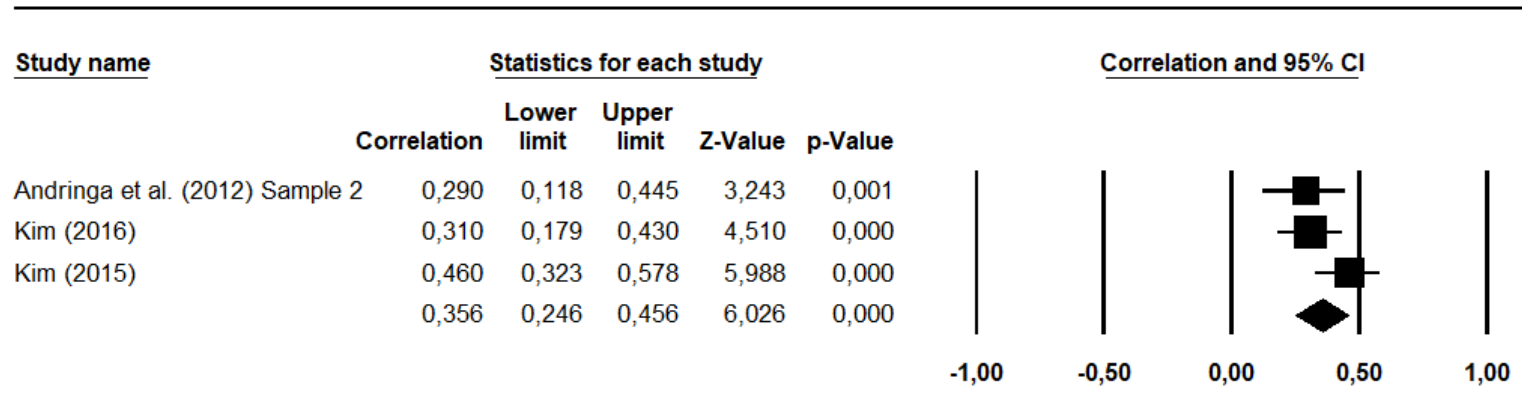

Figure 4. Reported correlations between grammar knowledge and listening comprehension

The overall correlation between L1 grammar and L1 listening comprehension was positive with medium effect size as well ( $r=.36,95 \%$ CI [.25-.46]) and the association was significant $(p<.001)$. It is clear that both linguistic factors have similar effects on the overall listening comprehension scores with a slight advantage of vocabulary knowledge over grammar knowledge.

\subsubsection{Morphological and phonological awareness and L1 listening comprehension}

There were only two studies (Fracasso et al., 2016; Lepola et al., 2012) addressing the relationship between phonological and morphological awareness and listening comprehension, with each component being addressed in one study. Phonological awareness had a small positive correlation with L1 listening comprehension $(r=.31$, 95\% CI [.15-.46]) and the interaction was significant $(\mathrm{p}<.001)$. On the other hand, morphological awareness displayed a significant $(\mathrm{p}<.001)$ and large $(r=.76,95 \%$ CI [.62-.85]) correlation with listening comprehension.

\subsubsection{Cognitive correlates of L1 listening comprehension}

The only cognitive component addressed by the studies conducted on L1 listening comprehension was working memory. Six correlations were obtained from six different studies with 750 participants (mean sample size $=125, S D=47.97$, range $=$ 66-201). Correlation coefficients are presented in Figure 5. 


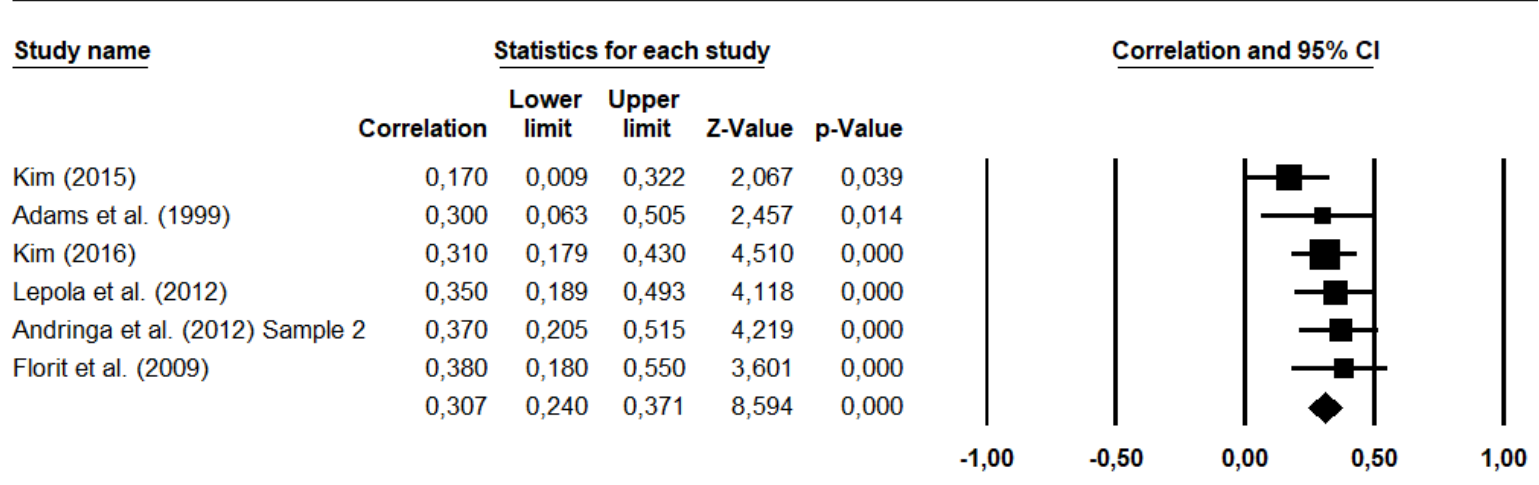

Figure 5. Reported correlations between working memory and listening comprehension

Working memory had a positive correlation with L1 listening comprehension with a small effect size $(r=.31,95 \%$ CI [.24-.37]) and the correlation was significant $(p<$ .001). This suggests that the role of cognition is relatively limited in L1 listening when compared to linguistic components.

\subsection{Correlates of L2 listening comprehension}

The number of independent samples, overall correlation coefficients and 95\% confidence of intervals of the correlates of L2 listening comprehension are presented in Table 3.

Table 3. Overall correlations between the components and L2 listening comprehension

\begin{tabular}{llll}
\hline Component & $\mathrm{N}$ & $r[95 \% \mathrm{CI}]$ & $I^{2}$ \\
\hline Vocabulary Size & 16 & $.62[.55-.68]^{* *}$ & 82.682 \\
Vocabulary Depth & 3 & $.80[.57-.91]^{* *}$ & 92.933 \\
Grammar Knowledge & 3 & $.69[.54-.79]^{* *}$ & 71.560 \\
Working Memory & 10 & $.30[.24-.35]^{* *}$ & 29.243 \\
Metacognition & 7 & $.54[.29-.72]^{* *}$ & 93.804 \\
Self-concept & 4 & $.55[.34-.70]^{* *}$ & 88.053 \\
Anxiety & 4 & $-.59[-.73--.42]^{* *}$ & 86.678 \\
\hline$* * p<.001$ & & &
\end{tabular}

Table 3 underlines that the mostly studied correlate of L2 listening comprehension is vocabulary knowledge followed by working memory and metacognition. Unlike the results obtained in L1 studies, the studies reported large effect sizes, especially in linguistic components. Additionally, affective components were also reported to have correlated with L2 listening comprehension, which was not the case in L1 studies.

\subsubsection{Linguistic correlates of L2 listening comprehension}

\subsubsection{Vocabulary knowledge and L2 listening comprehension}

Vocabulary knowledge was investigated through 14 studies with 16 independent samples and 2218 participants from several linguistic backgrounds including Chinese (4 samples), Turkish (1 sample), Danish (2 samples), Japanese (1 sample), Norwegian 
(1 sample) and Swiss (1 sample) (mean sample size $=158.42, S D=75.44$, range $=59$ 354). Among the participants, one sample learned Dutch, one learned German, and the rest learned English as their L2. The studies and their reported correlations between vocabulary knowledge and L2 listening comprehension are presented in Figure 6.

\begin{tabular}{|c|c|c|c|c|c|c|c|c|}
\hline \multirow[t]{2}{*}{ Study name } & \multicolumn{4}{|c|}{ Statistics for each study } & \multirow[b]{2}{*}{ p-Value } & \multicolumn{3}{|c|}{ Correlation and $95 \% \mathrm{CI}$} \\
\hline & Correlation & $\begin{array}{l}\text { Lower } \\
\text { limit }\end{array}$ & $\begin{array}{l}\text { Upper } \\
\text { limit }\end{array}$ & Z-Value & & & & \\
\hline Bonk (2000) & 0,440 & 0,207 & 0,626 & 3,534 & 0,000 & & & \\
\hline Wang \& Treffers-Daller (2017) & 0,440 & 0,311 & 0,553 & 6,139 & 0,000 & & & \\
\hline Tighe et al. (2015) Sample 3 & 0,460 & 0,337 & 0,567 & 6,654 & 0,000 & & & \\
\hline Vulchanove et al. (2014) & 0,460 & 0,272 & 0,614 & 4,476 & 0,000 & & & \\
\hline Wolfgramm et al. (2016) & 0,480 & 0,396 & 0,556 & 9,798 & 0,000 & & & \\
\hline Vandergrift \& Baker (2015) & 0,510 & 0,384 & 0,617 & 6,983 & 0,000 & & & \\
\hline Tighe et al. (2015) Sample 2 & 0,570 & 0,465 & 0,659 & 8,807 & 0,000 & & & \\
\hline Oh (2016) & 0,590 & 0,419 & 0,720 & 5,750 & 0,000 & & & \\
\hline Tighe et al. (2015) Sample 1 & 0,620 & 0,530 & 0,696 & 10,556 & 0,000 & & & \\
\hline Andringa et al. (2012) & 0,680 & 0,566 & 0,768 & 8,696 & 0,000 & & & \\
\hline Stæhr (2008) & 0,690 & 0,562 & 0,786 & 7,818 & 0,000 & & & \\
\hline Stæhr (2009) & 0,700 & 0,593 & 0,783 & 9,179 & 0,000 & & & \\
\hline Teng (2016) & 0,700 & 0,575 & 0,793 & 7,996 & 0,000 & & & \\
\hline Saglam (2014) & 0,710 & 0,574 & 0,808 & 7,423 & 0,000 & & & \\
\hline Matthews \& Cheng (2015) & 0,730 & 0,650 & 0,794 & 11,894 & 0,000 & & & \\
\hline \multirow[t]{3}{*}{ Teng (2014) } & 0,860 & 0,793 & 0,906 & 11,924 & 0,000 & & & \\
\hline & 0,617 & 0,549 & 0,677 & 13,603 & 0,000 & & & \\
\hline & & & & & & $-1,00$ & $-0,50$ & 0,50 \\
\hline
\end{tabular}

Figure 6. Reported correlations between vocabulary knowledge and listening comprehension

'I'he overall correlation between vocabulary knowledge and L' listening comprehension was found to be positive and the effect size was large $(r=.62,95 \% \mathrm{CI}$ [.55-.68]) and significant $(p<.001)$. This indicates that vocabulary knowledge, as measured through vocabulary size tests or recognition tests, was a strong indicator of L2 listening comprehension. Higher vocabulary knowledge scores brought about higher scores on listening comprehension tasks.

In addition to vocabulary size, depth of vocabulary knowledge was also investigated through three independent samples of 291 participants in total. Two samples had Chinese as their L1s and one sample came from Danish background. Figure 7 presents the studies and the reported correlations between the depth of vocabulary knowledge and listening comprehension.

\begin{tabular}{|c|c|c|c|c|c|c|c|c|c|}
\hline \multirow[t]{2}{*}{ Study name } & \multirow[b]{2}{*}{ Correlation } & \multicolumn{3}{|c|}{ Statistics for each study } & \multirow[b]{2}{*}{ p-Value } & & \multicolumn{3}{|c|}{ Correlation and $95 \% \mathrm{Cl}$} \\
\hline & & $\begin{array}{l}\text { Lower } \\
\text { limit }\end{array}$ & $\begin{array}{c}\text { Upper } \\
\text { limit }\end{array}$ & Z-Value & & & & & \\
\hline Stæhr (2009) & 0,650 & 0,530 & 0,744 & 8,205 & 0,000 & & & & \\
\hline Teng (2016) & 0,750 & 0,641 & 0,829 & 8,970 & 0,000 & & & & \\
\hline \multirow[t]{2}{*}{ Teng (2014) } & 0,910 & 0,866 & 0,940 & 14,083 & 0,000 & & & & \\
\hline & 0,797 & 0,571 & 0,911 & 4,842 & 0,000 & & & & \\
\hline
\end{tabular}

Figure 7. Reported correlations between the depth of vocabulary knowledge and listening comprehension 
The overall correlation between the depth of vocabulary knowledge and L2 listening comprehension was positive and the effect size of the association was large $(r=.79$, $95 \%$ CI [.57-.91]) and significant $(p<.001)$.

This finding, along with that of vocabulary size, suggests that both dimensions of vocabulary knowledge are important contributors to L2 listening comprehension and that vocabulary size should be reinforced with the depth of vocabulary knowledge.

\subsubsection{Grammar knowledge and L2 listening comprehension}

Another linguistic component addressed in L2 listening studies was grammar knowledge. Correlation results for this component came from three different samples, as in the case of L1 studies, with 261 participants in total (mean sample size $=87, S D$ $=22.53$, range $=73-113$ ). Overall correlation coefficients and 95\% confidence of intervals for the association between grammar knowledge and L2 listening comprehension are presented in Figure 8.

\begin{tabular}{lrllrrr} 
Study name & \multicolumn{6}{c}{ Statistics for each study } \\
\cline { 2 - 6 } & Correlation & $\begin{array}{c}\text { Lower } \\
\text { limit }\end{array}$ & $\begin{array}{c}\text { Upper } \\
\text { limit }\end{array}$ & Z-Value & p-Value \\
Oh (2016) & 0,550 & 0,369 & 0,691 & 5,247 & 0,000 \\
Saglam (2014) & 0,700 & 0,560 & 0,801 & 7,256 & 0,000 \\
Andringa et al. (2012) & 0,770 & 0,682 & 0,836 & 10,701 & 0,000 \\
& 0,687 & 0,543 & 0,792 & 7,038 & 0,000
\end{tabular}

Figure 8. Reported correlations between grammar knowledge and listening comprehension

There exists a positive overall correlation between grammar knowledge and L2 listening comprehension with a large effect size $(r=.69,95 \%$ CI [.54-.79]) and the association between the two is significant $(p<.001)$. Although there is a scarcity of studies conducted in this domain compared to vocabulary knowledge, the results reveal that grammar is also a significant predictor of L2 listening comprehension success. As in the case of vocabulary knowledge, higher scores on grammar tests were accompanied by higher listening comprehension scores. Taken together, vocabulary and grammar knowledge yielded stronger correlations with L2 listening comprehension than with L1 listening comprehension scores.

\subsubsection{Cognitive correlates of L2 listening comprehension}

In addition to linguistic components which depend on the linguistic proficiency of learners, correlations of language-neutral components were also reported which are independent of language proficiency. These correlates were working memory and metacognition (as measured by strategic listening skills and metacognitive awareness tests). 


\subsubsection{Working memory and L2 listening comprehension}

There were correlation reports from eight studies and ten independent samples with 1522 participants coming from varying linguistic backgrounds (mean sample size $=190.25, S D=89.56$, range $=41-354)$. Individual and overall correlations along with their $95 \%$ confidence of interval scores are shown in Figure 9.

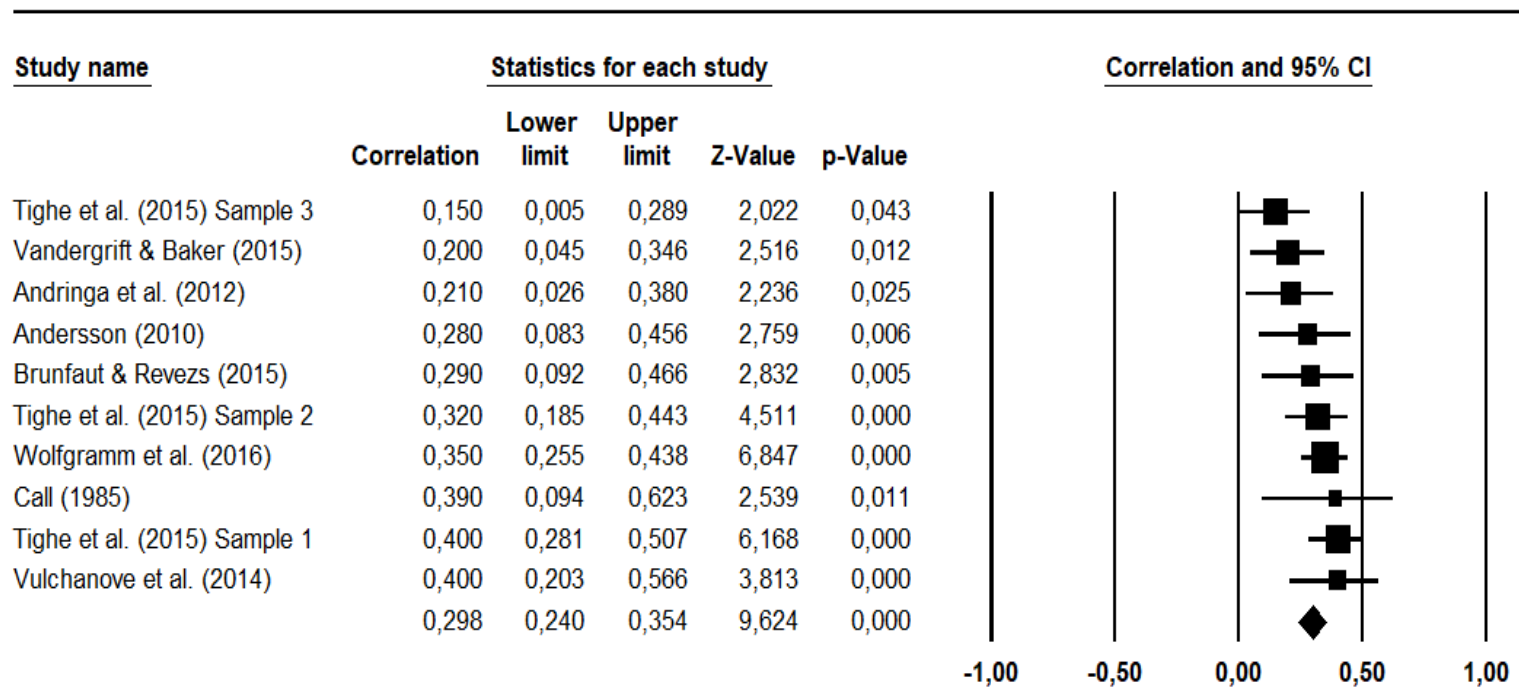

Figure 9. Reported correlations between working memory and listening comprehension

Overall correlation between working memory and L2 listening comprehension was small $(r=.30,95 \%$ CI [.24-.35]) and significant $(p<.001)$. It is demonstrated by the results that working memory was not as significant a factor in explaining L2 listening comprehension as linguistic factors of grammar and vocabulary knowledge.

A similar case was observed in L1 listening comprehension with working memory yielding smaller correlations with comprehension than linguistic components of vocabulary and grammar knowledge. Taken together with the results obtained in L1 listening comprehension, the results highlight the superiority of linguistic components over working memory in terms of their contribution to listening comprehension.

\subsubsection{Metacognition and L2 listening comprehension}

Unlike studies on L1 listening comprehension, those investigating L2 listening comprehension reported correlation results for another cognitive component, metacognition. The results were obtained from seven studies with seven independent samples and 725 subjects (mean sample size $=103.57, S D=46.7$, range $=44-172$ ). Figure 10 presents the individual and collective correlation scores between metacognition and L2 listening comprehension.

As demonstrated in Figure 10, correlation between metacognition and L2 listening comprehension is positive with a large $(r=.54,95 \%$ CI [.29-.72]) and statistically significant effect size $(p<.001)$. Metacognition was found to be a far more important determiner of L2 listening comprehension success than the other cognitive component, working memory. 


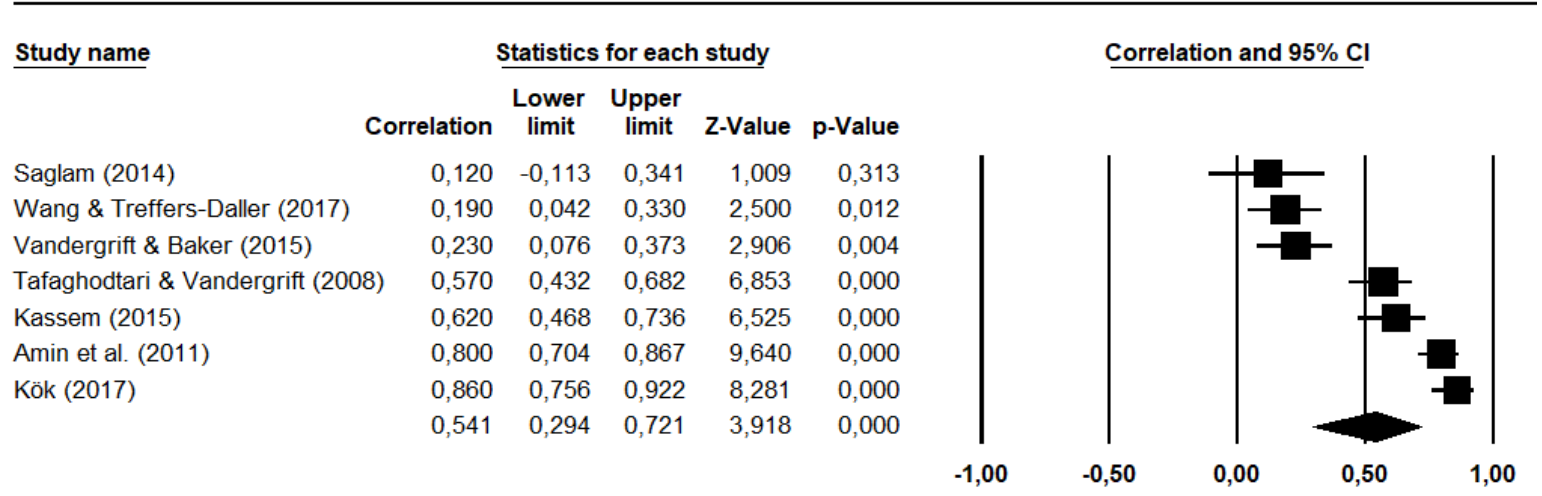

Figure 10. Reported correlations between metacognition and listening comprehension

\subsubsection{Affective correlates of L2 listening comprehension}

An important discovery in the current meta-analysis is the strong association between affective factors and L2 listening comprehension. This was not the case with L1 listening comprehension since no studies reported such correlations. Affective correlates consisted of self-concept (motivation and self-efficacy) and anxiety (foreign language listening anxiety, and foreign language learning anxiety).

\subsubsection{Self-concept and L2 listening comprehension}

Four studies with four independent samples and 620 participants reported correlations between self-concept and L2 listening comprehension (mean sample size $=155, S D=134.48$, range $=61-354)$. The results are presented in Figure 11.

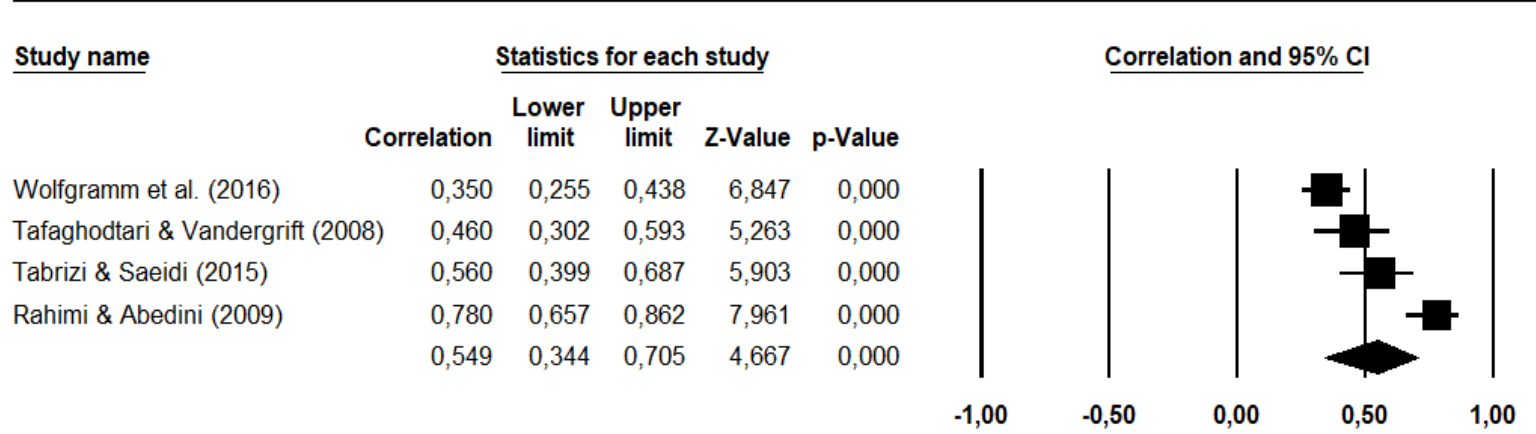

Figure 11. Reported correlations between self-concept and listening comprehension

There was a positive correlation between self-concept and L2 listening comprehension with a large effect size $(r=.55,95 \%$ CI [.34-.70]) and the relationship between the two was significant $(p<.001)$. This indicates that learners' motivation towards and perceptions of success in the target language play an important role in listening comprehension scores. However, the relationship between these two may be in the other direction; high performance on listening may have brought about higher perceptions of success and motivation in the target language. 


\subsubsection{Anxiety and L2 listening comprehension}

Another recurring affective contributor to listening comprehension was anxiety and four correlation reports came from four studies with four samples and 596 participants in total (mean sample size $=149, S D=85.31$, range $=60-233$ ). The results are displayed in Figure 12.

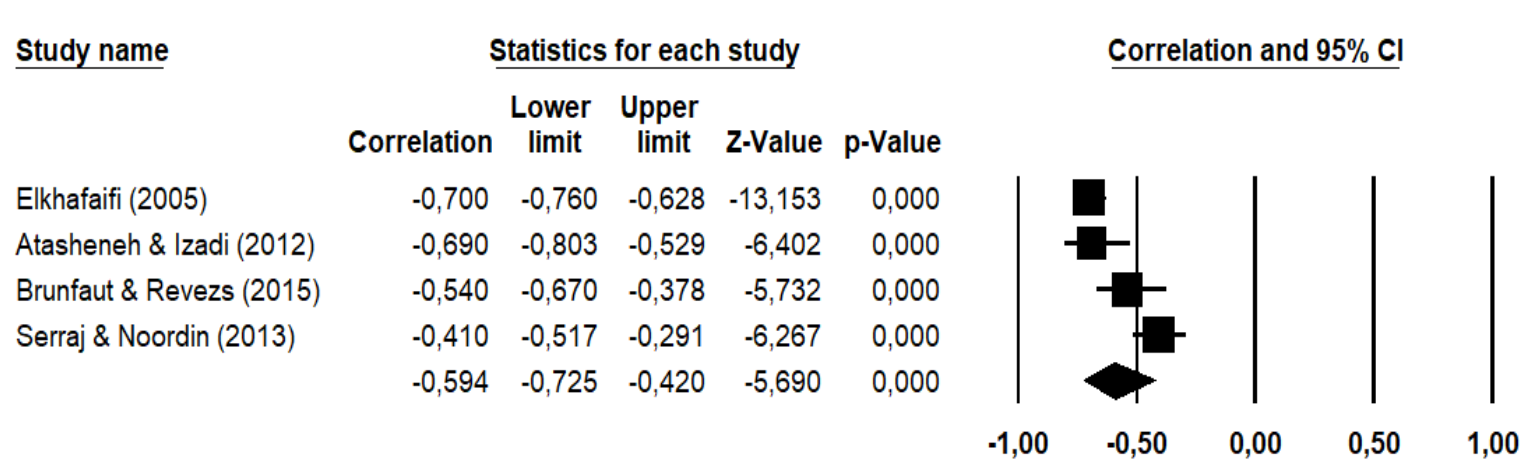

Figure 12. Reported correlations between anxiety and listening comprehension

There was a negative correlation between anxiety and L2 listening comprehension and the effect size was large $(r=-.59,95 \%$ CI [-.73--.42]) and significant $(p<.001)$. Unlike the rest of the correlates of listening comprehension, anxiety had a negative relationship with comprehension, meaning that lower anxiety scores brought about higher comprehension scores.

Similar to the other affective component, the relationship between the two may be in the other direction; higher performance in listening comprehension may have brought about lower levels of anxiety.

\section{Discussion}

The results of the current meta-analysis underlined some important points: (a) insufficient empirical accumulation on listening skill, (b) the need for adopting a componential approach to listening comprehension, (c) similarities between listening and reading comprehension in terms of the components involved in the process and $\mathrm{d}$ ) similarities/differences between L1 and L2 listening comprehension.

Limiting the discussion to studies with a correlational design, it is obvious from the number of studies on listening comprehension that listening is a neglected skill. A similar meta-analysis by Jeon and Yamashita (2014) conducted on the components of L2 reading comprehension alone included 59 studies while the present meta-analysis was based on only 33 studies including L1 and L2 contexts together. Besides, for low power of analysis concerns, no moderator variables (age, gender, L1, proficiency, tests used to measure each component) could be investigated due to insufficient empirical studies present up to the present time. In this sense, it might be argued that listening comprehension studies are at their infancy and the area needs further empirical evidence. Therefore, the discussions put forward here should be taken with great care. 
Although there is a scarcity of studies addressing listening comprehension, there are some significant findings in the previous research. First, listening comprehension in L1 and L2 was found to be correlating with several factors, which requires to take a componential view to listening skills. Construction Integration Model by Kintsch (1988) advocates that comprehension of texts is a complex multi-layered process dependent on both linguistic knowledge and cognitive skills and the results of the current meta-analysis provide evidence on the complex nature of listening skill with correlation reports between cognitive, linguistic and affective factors, and listening comprehension. In this regard, we can argue that studies addressing listening comprehension should take into consideration at least these three domains to provide a comprehensive picture of oral comprehension. However, it is important to bear in mind that this meta-analysis was limited in its scope as it focused on studies with correlational design. As a result, it is conceivable that there might be several other contributors to listening comprehension addressed by studies with different methodological designs (e.g., experimental). Hence, it is important to investigate listening comprehension within a componential approach and to take into consideration multiple component skills in explaining success in this area. In addition to researchers, practitioners are also to take into account as many component skills as possible and to develop component skills first to improve listening comprehension among language learners. The results of the current meta-analysis in this sense can be an important guide to detect some of the areas to improve for promoting listening comprehension.

Another important finding is the close similarity between L2 listening and L2 reading comprehension as two receptive skills. The similarity between the two was related to the components involved and their strengths of association with comprehension. A similar meta-analysis by Jeon and Yamashita (2014) showed that vocabulary and grammar knowledge were two key predictors of reading comprehension. This might stem from the fact that both skills are receptive (Davies, 1976) and include similar processes to comprehend the incoming messages by attending to the linguistic features including syntactic and semantic properties of a given text. Thence, processing the incoming messages, either spoken or written, would require learners to employ their vocabulary and grammar knowledge together. Our results also underscore the important role vocabulary and grammar knowledge play in determining listening comprehension scores. Similar to L2 reading studies which report strong links between vocabulary knowledge and comprehension (Hawas, 1990; Khaldieh, 2001; Koda, 1992; Pulido \& Hambrick, 2008; Shiotsu, 2010), strong associations were found between vocabulary knowledge and L2 listening comprehension (Matthews \& Cheng, 2015; Sağlam, 2014; Stæhr, 2009; Teng, 2014, 2016). It is strongly advocated that core word knowledge should be promoted to enable learners to comprehend texts (Koda, 2005). This view is well reflected in both L2 reading and listening comprehension studies with the majority of studies addressing this component much more frequently than any other factor. The role of vocabulary knowledge is almost taken for granted, and as a result, most studies ended up 
focusing on vocabulary knowledge over other linguistic components, especially grammar knowledge, in investigating L2 listening and reading skills. However, defined as "the knowledge about a variety of morphosyntactic properties such as tense, aspect, word order, subject-verb agreement, and articles" (Jeon \& Yamashita, 2014 , p. 165), grammar knowledge proved to be as strong as, if not stronger, than vocabulary knowledge in explaining variance in L2 listening (Andringa et al., 2012; Sağlam, 2014) and reading comprehension (Bensoussan \& Ramraz, 1984; Lefrancois \& Armand, 2003; Shiotsu \& Weir, 2007). Considering that grammar knowledge enables readers and listeners to integrate word knowledge and syntactic knowledge at the phrase, clause and sentence levels (Jeon \& Yamashita, 2014), it can be argued that these two component skills go hand in hand together. Therefore, studies should not ignore the important role of grammar knowledge for the sake of vocabulary knowledge. We can then extend Koda's (2005) suggestion that vocabulary knowledge is prerequisite for reading comprehension to grammar knowledge and to listening contexts as well. Finally, and perhaps most importantly, both reading and listening comprehension were found to benefit more from linguistic components than cognitive components. This finding is significant as it underlines the importance of linguistic development in explaining text comprehension. Alderson (1984) asked a commonly addressed question of whether L2 reading comprehension is a language or a reading problem. Converting the question into listening comprehension and asking whether listening comprehension is a language or a listening problem, the answer derived from the current meta-analysis would be that listening comprehension is a linguistic problem rather than a listening problem on its own. In other words, working memory, which is independent of linguistic proficiency, has less effect on listening comprehension than vocabulary and grammar knowledge. The relatively limited effect of working memory on both L2 listening and reading comprehension as well as the fact that working memory is a component beyond the reach of teachers thus impossible to intervene, highlight the significance of linguistic components in determining variance among individuals. Therefore, the suggestion that grammar and vocabulary knowledge should be promoted first seems to be a valid one.

An important contribution of the current meta-analysis to literature is the comparison between L1 and L2 listening comprehension in terms of the factors involved in the process. This comparison would allow us to determine whether the process is dependent on the status of the target language. To this end, noteworthy similarities and differences were found between L1 and L2 listening comprehension. Similar to the resemblance of listening to reading comprehension, linguistic components had stronger correlations to comprehension than working memory in both L1 and L2 listening comprehension. This is also strong evidence of comprehension being a matter of linguistic knowledge rather than a listening problem on its own. This finding is an indicator of the fact that even the native speakers, assumedly more competent speakers of the language, are vulnerable to differences in linguistic components in the process of text comprehension. Then it is the responsibility of the teachers and practitioners to develop linguistic components first before expecting 
language learners to comprehend the texts brought to language classrooms. It should be kept in mind that what causes the students to fail might be the linguistic deficiencies rather than deficiencies in listening skills.

Finally, there were significant differences between L1 and L2 listening comprehension. The first one is related to the predictive value of linguistic components in explaining comprehension. A close inspection of the results demonstrates that the effect of grammar and vocabulary knowledge was smaller in native listening comprehension than in L2 listening comprehension. It might be because of the fact that L1 speakers don't demonstrate great differences in their grammar and vocabulary knowledge of their mother tongue, resulting in small effect sizes of these two components in their overall comprehension success. Unlike L1 speakers, L2 learners were more sensitive to differences in these two component skills because of the probable individual differences in linguistic proficiency. A more detailed inspection of the two linguistic components underlined that grammar knowledge was less effective than vocabulary knowledge in explaining success in L1 listening comprehension. Considering that there are limited grammatical patterns in a language but far more lexical knowledge to be acquired, it is expected that the natives have reached near perfection in their grammar but their lexical growth is in constant progress. Therefore, while grammar knowledge is expected to be at a certain level for all native speakers, vocabulary knowledge might show much more variance from individual to individual. As a result, grammar and vocabulary knowledge shows differential effects on L1 and L2 listening.

The second major difference between L1 and L2 listening comprehension is the effect of metacognition on the latter. Defined as conscious and goal-oriented procedures facilitating a learning task (Chamot, 2005), learning strategies yielded strong correlations with L2 listening comprehension meaning that it is an important predictor of success in L2 listening comprehension. Also, previous experimental studies seeking to find out the effect of learning strategy instruction on listening comprehension (Carrier, 2003; Ross \& Rost, 1991; Thompson \& Rubin, 1996) clearly demonstrated that such instruction led to significant improvements in oral comprehension. Taking into consideration the experimental evidence and the strong correlations obtained from our study, we can argue that metacognition is an important component of listening comprehension in L2. The difference between L1 and L2 in this aspect may be resulting from the fact that L1 listening does not pose a learning task for native speakers as L2 does for language learners. Consequently, it is suggested for scholars that they take into consideration metacognition as part of L2 studies and the practitioners that they deliver listening comprehension strategy instruction to improve L2 listening comprehension.

Another striking difference is related to correlations between affective components and listening comprehension. While L1 listening comprehension is not reported to correlate with any affective factors, moderate to strong correlations were found between L2 listening comprehension and self-concept (as measured by motivation and self-efficacy scores) and anxiety. As Oxford and Shearin (1994) suggest, motivation is 
directly associated with how much effort an L2 learner would invest in learning the target language and how much they would engage in language learning tasks in and out of class and how often they would use language learning strategies. Considering this broad range of motivation influences, it is not surprising to see reports of its strong correlation with L2 listening. Similarly, learners' beliefs about their capabilities to regulate their own learning determine their motivation levels and academic accomplishments as well (Bandura, 1993). Self-efficacy in this sense may contribute to L2 listening either directly or indirectly by increasing the level of motivation to learn the language. Further studies are suggested to test this but what is noteworthy is the large contribution of these factors to comprehension. Unlike motivation and self-efficacy beliefs, anxiety correlated negatively with L2 listening comprehension. In a review of studies addressing language learning anxiety, MacIntyre and Gardner (1991) underline that anxiety is an important problem that can have negative effects on the acquisition and production of the target language. This view was supported by the studies included in this meta-analysis. Based on the findings, it could be asserted that the affective domain has much to do with L2 listening comprehension. To this end, the model by Kintsch (1988) seems to be lacking in fully capturing the dynamics involved in text comprehension. The model involves cognitive, linguistic and general world knowledge components but disregards the affective domain. This may be due to the fact that the model was suggested for L1 comprehension. If we are to apply this model to L2 text comprehension, we should take into consideration the suggestion by Krashen (1985) that L2 learners bring with themselves "affective filters" and add an affective component to the model. Affective components as suggested by the findings can comprise of learners' motivation towards and their beliefs about their performance in the target language and their anxiety levels in the language classroom. However, it should be noted that the relation between these components may be bidirectional. Low level of success in listening may lead to the low level of self-concept and high levels of anxiety or low level of success in listening comprehension may lead to the low level of self-concept and high level of anxiety. In this sense, affective factors may not be necessarily the components but the mediators, or broadly speaking, correlates of L2 listening comprehension.

To summarize, listening comprehension in L1 and L2 is a complex process involving linguistic, cognitive and affective correlates. Especially considering the important role listening texts brought to teaching environments play in developing the learners' academic and linguistic skills (Rashidi \& Khosravi, 2010), it is a must for researchers and practitioners alike to cater for as many factors as possible to decrease the effect of individual differences among language learners on L2 listening comprehension.

\section{Conclusions}

The present meta-analysis set out to (a) investigate the correlates of L1 and L2 listening comprehension and (b) discover their strengths of association with L1 and L2 listening comprehension and (c) find out the similarities and differences between L2 listening and reading comprehension. To this end, primary studies reporting 
correlation results between listening comprehension and its components were retrieved from several databases. A total of 33 studies (26 studies addressing L2, 7 studies addressing L1 listening comprehension) reported correlation scores between linguistic, cognitive and affective factors and listening comprehension. As a result of insufficient empirical evidence, the results obtained in this meta-analysis should be taken with great care.

Despite the low number of studies, the combined correlation scores from the studies under review yielded several significant arguments for discussion. First and most important of all, it was clearly seen that like reading comprehension, listening comprehension is a complex skill comprised of several components. The studies under review showed that listening comprehension could be explained through success in linguistic, cognitive and affective factors. This requires researchers and teachers to take a componential to listening comprehension and to improve component skills for developing listening comprehension.

Second, it was found that linguistic components, grammar and vocabulary knowledge in the present case, were more strongly associated with listening comprehension in L1 and L2 than working memory. This indicated that comprehension is more of a matter of linguistic knowledge than cognition. The results in this sense are similar to those obtained in reading comprehension. Grammar and vocabulary were found to be correlating with reading comprehension significantly more strongly than working memory.

Another significant finding was the difference between L1 and L2 listening comprehension in terms of the components involved and their strengths of association with comprehension. Unlike L1 listening comprehension, L2 listening comprehension was sensitive to metacognition and affective components such as motivation and anxiety. While there were no reports of a correlation between metacognition, affect and listening comprehension in L1, such scores were commonly observed in L2 contexts, which underlined the need to take into consideration the affective factors in language classrooms. Higher levels of motivation and self-efficacy along with lower anxiety levels brought about higher scores in listening comprehension.

The oft-discussed problem that listening is a neglected skill is evident from the number of studies included in this meta-analysis. In parallel with this insufficient empirical accumulation and a limited number of participants included, the effect of moderator variables such as the participants' age, the tasks being receptive or productive, L1 and L2 distance could not be investigated. Therefore, the results reported here represent heterogeneous samples, which discourage making strong claims about the correlates of listening comprehension. Therefore, further studies are needed to add to our understanding of L1 and L2 comprehension.

In addition to insufficient empirical data, this meta-analysis is also limited in that only those studies reporting correlation scores were included in the analysis with experimental studies being excluded. Consequently, a further meta-analysis of 
experimental studies should be conducted to capture a more comprehensive picture of the components involved in the listening comprehension process.

\section{References}

(References with an asterisk were included in the meta-analysis)

*Adams, A. M., Bourke, L., \& Willis, C. (1999). Working memory and spoken language comprehension in young children. International Journal of Psychology, 34, 364-373. http://dx.doi.org/ 10.1080/002075999399701

*Amin, I. A. R., Aly, M. A. S., \& Mohammed, A. M. (2012). A correlation study between EFL strategic listening and listening comprehension skills among secondary school students. Benha Faculty of Education Journal, 23(1), 1-26.

Alderson, J. C. (1984). Reading in a foreign language: A reading problem or a language problem? In J. C. Alderson \& A. H. Urquhart (Eds.), Reading in a foreign language (pp. 124). London: Longman.

Al Seyabi, F., \& Tuzlukova, V. (2014). Writing Problems and Strategies: An Investigative Study in the Omani School and University Context. Asian Journal of Social Sciences \& Humanities, 3(4), 37-48.

*Andersson, U. (2010). The contribution of working memory capacity to foreign language comprehension in children. Memory, 18, 458-472. http://dx.doi.org/ 10.1080/09658211003762084

*Andringa, S., Olsthoorn, N., van Beuningen, C., Schoonen, R., \& Hulstijn, J. (2012). Determinants of success in native and non-native listening comprehension: An individual differences approach. Language Learning, 62, 49-78. http://dx.doi.org/ 10.1111/j.14679922.2012.00706.x

*Atasheneh, N., \& Izadi, A. (2012). The role of teachers in reducing/increasing listening comprehension test anxiety: A case of Iranian EFL learners. English Language Teaching, 5, 178-187. http://dx.doi.org/ 10.5539/elt.v5n3p178

Bandura, A. (1993). Perceived self-efficacy in cognitive development and functioning. Educational Psychologist, 28, 117-148. http://dx.doi.org/ 10.1207/s15326985ep2802_3

Beal, V. (2007). The weight of involvement load in college level reading and vocabulary tasks (Doctoral dissertation). Retrieved from Concordia University CLUES Library Catalogue (LE 3 C66E38M 2007 B43)

Bensoussan, M., \& Ramraz, R. (1984). Testing EFL reading comprehension using a multiplechoice rational cloze. Modern Language Journal, 68, 230-239. http://dx.doi.org/ 10.1111/j.1540-4781.1984.tb01569.x

*Bonk, W. J. (2000). Second language lexical knowledge and listening comprehension. International Journal of Listening, 14, 14-31. http://dx.doi.org/ 10.1080/10904018.2000.10499033

*Brunfaut, T., \& Révész, A. (2015). The role of task and listener characteristics in second language listening. Tesol Quarterly, 49(1), 141-168.

${ }^{*}$ Call, M. E. (1985). Auditory short-term memory, listening comprehension, and the input hypothesis. TESOL Quarterly, 19, 765-781. http://dx.doi.org/ 10.2307/3586675

Carr, T. H., \& Levy, B. A., (Eds.) (1990). Reading and its development: Component skills approaches. San Diego: Academic Press.

Carrier, K. A. (2003). Improving high school English language learners' second language listening through strategy instruction. Bilingual Research Journal, 27, 383-408. http://dx.doi.org/ 10.1080/15235882.2003.10162600 
Chamot, A. U. (2005). Language learning strategy instruction: Current issues and research. Annual Review of Applied Linguistics, 25, 112-130. http://dx.doi.org/ $10.1017 / \mathrm{s} 0267190505000061$

Chen, K. Y. (2011). The impact of EFL students' vocabulary breadth of knowledge on literal reading comprehension. Asian EFL Journal, 51, 30-40.

Cheng, Y. S. (2002). Factors associated with foreign language writing anxiety. Foreign Language Annals, 35(6), 647-656.

Cheng, Y. S., Horwitz, E. K., \& Schallert, D. L. (1999). Language anxiety: Differentiating writing and speaking components. Language Learning, 49(3), 417-446.

Choi, H. Y. (2013). Effects of depth and breadth of vocabulary knowledge on English reading comprehension among Korean high school students. Language Research, 49, 419-452.

Davies, N. F. (1976). Receptive versus productive skills in foreign language learning. The Modern Language Journal, 60, 440-443. http://dx.doi.org/ 10.1111/j.15404781.1976.tb03667.x

*Elkhafaifi, H. (2005). Listening comprehension and anxiety in the Arabic language classroom. The Modern Language Journal, 89, 206-220. http://dx.doi.org/ 10.1111/j.15404781.2005.00275.x

*Florit, E., Roch, M., Altoè, G., \& Levorato, M. C. (2009). Listening comprehension in preschoolers: The role of memory. British Journal of Developmental Psychology, 27, 935951. http://dx.doi.org/ 10.1348/026151008x397189

*Fracasso, L. E., Bangs, K., \& Binder, K. S. (2016). The contributions of phonological and morphological awareness to literacy skills in the adult basic education population. Journal of Learning Disabilities, 49, 140-151. http://dx.doi.org/ 10.1177/0022219414538513

Hawas, H. M. (1990). Vocabulary and reading comprehension: An experimental study. International Review of Applied Linguistics, 87, 45-63.

Huy, N. T. (2015). Problems affecting learning writing skill of grade 11 at Thong Linh high school. Asian Journal of Educational Research, 3(2), 53-69.

Jeon, E. H., \& Yamashita, J. (2014). L2 Reading comprehension and its correlates: A meta-analysis. Language Learning, 64, 160-212. http://dx.doi.org/ 10.1111/lang.12034

Jones, S., Myhill, D., \& Bailey, T. (2013). Grammar for writing? An investigation of the effects of contextualised grammar teaching on students' writing. Reading and Writing, 26(8), 12411263.

*Kassem, H. M. (2015). The relationship between listening strategies used by Egyptian EFL college sophomores and their listening comprehension and self-efficacy. English Language Teaching, 8, 153. http://dx.doi.org/ 10.5539/elt.v8n2p153

Keating, G. D. (2008). Task effectiveness and word learning in a second language: The involvement load hypothesis on trial. Language Teaching Research, 12(3), 365-386.

Khaldieh, S. (2001). The relationship between knowledge of "Icraab," lexical knowledge, and reading comprehension of non-native readers of Arabic. Modern Language Journal, 85, 416-431. http://dx.doi.org/ 10.1111/0026-7902.00117

Khotimah, S. (2014). The use of problem based learning to improve students' speaking ability. ELT Forum: Journal of English Language Teaching, 3(1), 50-56.

Kim, J., \& Cho, Y. (2015). Proficiency effects on relative rules of vocabulary and grammar knowledge in second language reading. English Teaching, 70, 75-96. http://dx.doi.org/ 10.15858/engtea.70.1.201503.75

*Kim, Y. S. (2015). Language and cognitive predictors of text comprehension: Evidence from multivariate analysis. Child Development, 86, 128-144. http://dx.doi.org/ $10.1111 / c d e v .12293$ 
*Kim, Y. S. G. (2016). Direct and mediated effects of language and cognitive skills on comprehension of oral narrative texts (listening comprehension) for children. Journal of Experimental Child Psychology, 141, 101-120. http://dx.doi.org/ 10.1016/j.jecp.2015.08.003

*Kim, Y. S., \& Phillips, B. (2014). Cognitive correlates of listening comprehension. Reading Research Quarterly, 49, 269-281. http://dx.doi.org/ 10.1002/rrq.74

Kintsch, W. (1988). The use of knowledge in discourse processing: A construction-integration model. Psychological Review, 95, 163-182.

*Kök, İ. (2017). Relationship between listening comprehension strategy use and listening comprehension proficiency. International Journal of Listening, 32(3), 163-179. http://dx.doi.org/ 10.1080/10904018.2016.1276457

Koda, K. (1992). The effects of lower-level processing skills on FL reading performance: Implications for instruction. Modern Language Journal, 76, 502-512. http://dx.doi.org/ 10.1111/j.1540-4781.1992.tb05400.x

Koda, K. (2005). Insights into second language reading: A cross-linguistic approach. New York: Cambridge University Press.

Krashen, S. D. (1985). The input hypothesis: Issues and implications. London: Longman.

Lee, S. K. (2007). Effects of textual enhancement and topic familiarity on Korean EFL students' reading comprehension and learning of passive form. Language Learning, 57, 87118. http://dx.doi.org/ 10.1111/j.1467-9922.2007.00400.x

Leeser, M. J. (2007). Learner-based factors in L2 reading comprehension and processing grammatical form: Topic familiarity and working memory. Language Learning, 57(2), 229270. http://dx.doi.org/ 10.1111/j.1467-9922.2007.00408.x

Lefrancois, P., \& Armand, F. (2003). The role of phonological and syntactic awareness in second-language reading: The case of Spanish-speaking learners of French. Reading and Writing: An Interdisciplinary Journal, 16, 219-246. http://dx.doi.org/ 10.1023/a:1022874425314

*Lepola, J., Lynch, J., Laakkonen, E., Silvén, M., \& Niemi, P. (2012). The role of inference making and other language skills in the development of narrative listening comprehension in 4-6-year-old children. Reading Research Quarterly, 47, 259-282. http://dx.doi.org/ 10.1002/rrq.020

*Matthews, J., \& Cheng, J. (2015). Recognition of high frequency words from speech as a predictor of L2 listening comprehension. System, 52, 1-13.

Nation, I. S. P. (2001). Learning vocabulary in another language. Cambridge: Cambridge University Press.

${ }^{*}$ Oh, E. (2016). Comparative studies on the roles of linguistic knowledge and sentence processing speed in L2 listening and reading comprehension in an EFL tertiary setting. Reading Psychology, 37(2), 257-285.

Oxford, R., \& Shearin, J. (1994). Language learning motivation: Expanding the theoretical framework. The Modern Language Journal, 78, 12-28. http://dx.doi.org/ 10.1111/j.15404781.1994.tb02011.x

Pérez Manzanilla, I. S., \& Díaz Cabrera, K. M. (2014). Factors that may have an impact on advanced EFL students' speaking ability (Master's Thesis). Retrieved from http://cdigital.uv.mx/handle/123456789/35250

Plonsky, L., \& Oswald, F. L. (2012). How to do a meta-analysis. In A. Mackey \& S. M. Gass (Eds.), Research methods in second language acquisition: A practical guide (pp. 275295). London: Basil Blackwell.

Plonsky, L., \& Oswald, F. L. (2014). How big is "big"? Interpreting effect sizes in L2 research. Language Learning, 64(4), 878-912. 
Pulido, D., \& Hambrick, D. Z. (2008). The "virtuous" circle: Modeling individual differences in L2 reading and vocabulary development. Reading in a Foreign Language, 20, 164-190.

*Rahimi, A., \& Abedini, A. (2009). The interface between EFL learners' self-efficacy concerning listening comprehension and listening proficiency. Novitas-Royal, 3, 14-28.

Rashidi, N., \& Khosravi, N. (2010). Assessing the role of depth and breadth of vocabulary knowledge in reading comprehension of Iranian EFL learners. Journal of Pan-Pacific Association of Applied Linguistics, 14, 81-108.

Ross, S., \& Rost, M. (1991). Learner use of strategies in interaction: Typology and teachability. Language Learning, 41, 235-273. http://dx.doi.org/ 10.1111/j.1467-1770.1991.tb00685.x

*Sağlam, S. (2014). The role of vocabulary breadth, syntactic knowledge, and listening strategy use on listening comprehension. Route Educational and Social Science Journal, 1, 54-72.

Saito, Y., Garza, T. J., \& Horwitz, E. K. (1999). Foreign language reading anxiety. The Modern Language Journal, 83(2), 202-218.

Sawaki, Y., Quinlan, T., \& Lee, Y. W. (2013). Understanding learner strengths and weaknesses: Assessing performance on an integrated writing task. Language Assessment Quarterly, 10(1), 73-95.

Scovel, T. (1978). The effect of affect on foreign language learning: A review of the anxiety research. Language Learning, 28(1), 129-142.

Sellers, V. D. (2000). Anxiety and reading comprehension in Spanish as a foreign language. Foreign Language Annals, 33(5), 512-520.

*Serraj, S., \& Noordin, N. B. (2013). Relationship among Iranian EFL students' foreign language anxiety, foreign language listening anxiety and their listening comprehension. English Language Teaching, 6(5), 1-12.

Shiotsu, T. (2010). Components of L2 reading: Linguistic and processing factors in the reading test performances of Japanese EFL learners. New York: Cambridge University Press.

Shiotsu, T., \& Weir, C. J. (2007). The relative significance of syntactic knowledge and vocabulary breadth in the prediction of reading comprehension test performance. Language Testing, 24, 99-128. http://dx.doi.org/ 10.1177/0265532207071513

*Stæhr, L. S. (2008). Vocabulary size and the skills of listening, reading and writing. Language Learning Journal, 36, 139-152. http://dx.doi.org/ 10.1080/09571730802389975

*Stæhr, L. S. (2009). Vocabulary knowledge and advanced listening comprehension in English as a foreign language. Studies in Second Language Acquisition, 31, 577-607. http://dx.doi.org/ 10.1017/s0272263109990039

*Tabrizi, H. M., \& Saeidi, M. (2015). The relationship among Iranian EFL learners' selfefficacy, autonomy and listening comprehension ability. English Language Teaching, 8, 158-169. http://dx.doi.org/ 10.5539/elt.v8n12p158

*Tafaghodtari, M. H., \& Vandergrift, L. (2008). Second and foreign language listening: Unraveling the construct. Perceptual and Motor Skills, 107, 99-113. http://dx.doi.org/ 10.2466/pms.107.1.99-113

Tahir, S. Z. (2015). Improving students' speaking skill through voice chat at University of Iqra Buru. Journal of Modern Education Review, 5(3), 296-306.

*Teng, F. (2014). Assessing the depth and breadth of vocabulary knowledge with listening comprehension. PASAA: Journal of Language Teaching and Learning in Thailand, 48, 2956.

*Teng, F. (2016). An in-depth investigation into the relationship between vocabulary knowledge and academic listening comprehension. TESL-EJ, 20 (2), 1-17. Retrieved from http://www.teslej.org/wordpress/issues/volume20/ej78/ej78a5 
*Tighe, E. L., Spencer, M., \& Schatschneider, C. (2015). Investigating predictors of listening comprehension in third-, seventh-, and tenth-grade students: a dominance analysis approach. Reading Psychology, 36, 700-740. http://dx.doi.org/ 10.1080/02702711.2014.963270

Thompson, I., \& Rubin, J. (1996). Can strategy instruction improve listening comprehension? Foreign Language Annals, 29, 331-342. http://dx.doi.org/ 10.1111/j.19449720.1996.tb01246.x

Urquhart, A. H., \& Weir, C. J. (1998). Reading in a second language: Process, product, and practice. New York: Longman.

*Vandergrift, L., \& Baker, S. (2015). Learner variables in second language listening comprehension: An exploratory path analysis. Language Learning, 65, 390-416. http://dx.doi.org/ 10.1111/lang.12105

*Vulchanova, M., Foyn, C. H., Nilsen, R. A., \& Sigmundsson, H. (2014). Links between phonological memory, first language competence and second language competence in 10year-old children. Learning and Individual Differences, 35, 87-95. http://dx.doi.org/ 10.1016/j.lindif.2014.07.016

*Wang, Y., \& Treffers-Daller, J. (2017). Explaining listening comprehension among L2 learners of English: The contribution of general language proficiency, vocabulary knowledge and metacognitive awareness. System, 65, 139-150. http://dx.doi.org/ 10.1016/j.system.2016.12.013

*Wolfgramm, C., Suter, N., \& Göksel, E. (2016). Examining the role of concentration, vocabulary and self-concept in listening and reading comprehension. International Journal of Listening, 30, 25-46. http://dx.doi.org/ 10.1080/10904018.2015.1065746

Woodrow, L. (2006). Anxiety and speaking English as a second language. RELC Journal, 37(3), 308-328.

Yalin, S., \& Wei, T. (2011). The relative significance of vocabulary breadth and syntactic knowledge in the prediction of reading comprehension test performance. Chinese Journal of Applied Linguistics, 34, 113-126. http://dx.doi.org/ 10.1515/cjal.2011.028

Zhiping, D., \& Paramasivam, S. (2013). Anxiety of speaking English in class among international students in a Malaysian university. International Journal of Education and Research, 1(11), 1-16.

Zin, M. Z., \& Rafik-Galea, S. (2010). Anxiety and academic reading performance among Malay ESL learners. Journal of Pan-Pacific Association of Applied Linguistics, 14(2), 41-58.

\section{Copyrights}

Copyright for this article is retained by the author(s), with first publication rights granted to the Journal.

This is an open-access article distributed under the terms and conditions of the Creative Commons Attribution license (CC BY-NC-ND) (http://creativecommons.org/licenses/by-nc-nd/4.0/). 